\title{
Simulating wet gas-solid fluidized beds using coarse-grid CFD-DEM
}

\author{
M. Girardi ${ }^{\text {a }}$, S. Radl ${ }^{\mathrm{b}}$, S. Sundaresan ${ }^{\mathrm{a}, *}$ \\ a Department of Chemical and Biological Engineering, Princeton University, Princeton, NJ, United States \\ ${ }^{\mathrm{b}}$ Institute of Process and Particle Engineering, Graz University of Technology, Graz, Austria
}

\section{H I G H L I G H T S}

- CFD-DEM simulations of wet gassolid fluidized beds in periodic domains have been performed.

- Surface tension force due to liquid bridges leads to agglomeration.

- Stronger cohesion requires a greater gas velocity to fully support the bed.

- Filtered drag coefficients are used in coarse-grid simulations of fluidized beds.

\section{A R T I C L E I N F O}

\section{Article history:}

Received 16 September 2015

Received in revised form

17 December 2015

Accepted 7 January 2016

Available online 29 January 2016

Keywords:

Multiphase flow

Fluidization

Filtering

Liquid bridges

Agglomeration

Numerical simulation
G R A P H I C A L A B S T R A C T

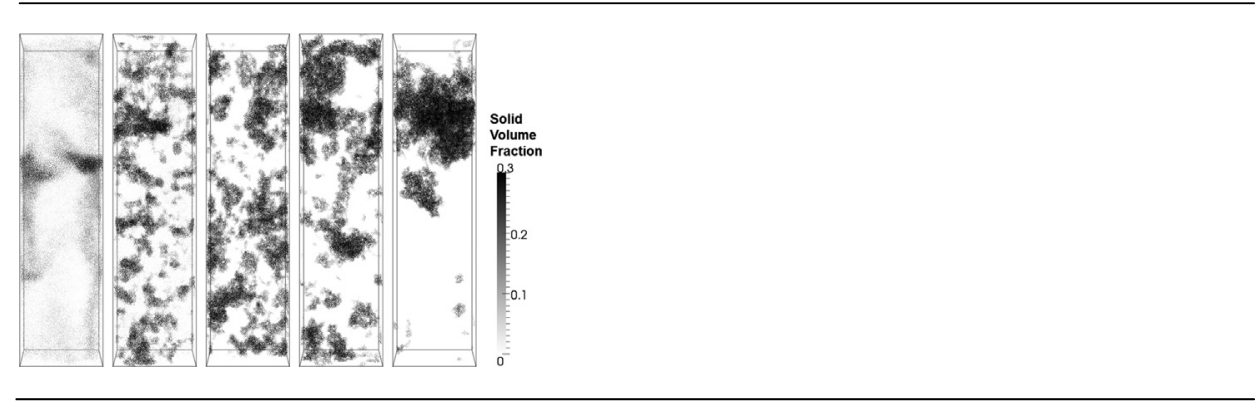

\begin{abstract}
A B S T R A C T
Simulations of wet fluidized beds of particles in small periodic domains have been carried out using a CFD-DEM approach. A liquid bridge forms upon particle-particle collision, which then ruptures when the particle separation exceeds a critical distance. The simulations take into account only the surface tension force of attraction due to the liquid bridge. Increasing the strength of cohesion leads to larger agglomerates, and correspondingly, higher gas velocities are required to fully support the particles. The slip velocity results from the simulations have been correlated in terms of a Bond number characterizing the strength of cohesion, volume of liquid in the bridge, and particle volume fraction. The CFD-DEM results are systematically coarse-grained to expose the dependence of the filtered drag coefficient on Eulerian filter size, surface tension forces, liquid loading, and solids loading in wet gas-solid fluidized beds.
\end{abstract}

(c) 2016 Elsevier Ltd. All rights reserved.

\section{Introduction}

Wet granular flows and fluidization of wet particles by a gas are important in a wide range of industrial processes, particularly in the energy and pharmaceutical industries (Muzzio et al., 2002). Liquid is often injected into gas-fluidized beds of particles that are used to carry out catalytic and non-catalytic reactions, drying, and granulation; examples include liquid gas-oil injection at the bottom of fluid catalytic crackers (Arbel et al., 1995), liquid injection in Wurster coaters (Christensen and Bertelsen, 1997; Shelukar, 2000;

\footnotetext{
* Corresponding author. Tel.: +1 609258 4583; fax.: +1 6092580211.

E-mail address: sundar@princeton.edu (S. Sundaresan).
}

Rajniak et al., 2009), granulators for the production of large agglomerates (Hapgood et al., 2007; Fries et al., 2011, 2013), and liquid bitumen injection in fluid cokers (Gray, 2002; Darabi et al., 2010).

Gas-fluidized beds of dry particles exhibit complex behavior, with instabilities giving rise to inhomogeneities that span a wide range of time and length scales (Sundaresan, 2000, 2003). Adding liquid to the system such that particles support a liquid film coating adds further complexity to the system. The liquid film coating allows for the formation of liquid bridges during particle-particle and particle-wall contacts, which may then lead to agglomeration (Herminghaus, 2005). Agglomerates complicate the flow structure, impeding smooth fluidization and causing slumping. Furthermore, 
the liquid may evaporate and react, producing large volumes of gas or solidifying otherwise shearable agglomerates. Overall, the presence of the liquid coating on particles has the potential to modify mass and heat transfer within the bed while also giving rise to altered secondary flow profiles and agglomeration. The effect of liquid on the fluidization characteristics has been investigated by several researchers (Seville and Clift, 1984; Seville, 1987; Passos and Mujumdar, 2000; McLaughlin and Rhodes, 2001; Wormsbecker and Pugsley, 2008; Darabi et al., 2011). Cohesive interaction between particles due to liquid bridges changes the nature of fluidization; upon increasing cohesion, Geldart group B particles manifest fluidization/defluidization characteristics that are reminiscent of group A particles, and with further increase in cohesion act as group $C$ particles (McLaughlin and Rhodes, 2001; Wormsbecker and Pugsley, 2008; Seville and Clift, 1984). Furthermore, the minimum fluidization velocity, which is typically defined as the gas velocity at which the pressure drop across the bed of particles exactly balances the weight of the particles, increases with strength of cohesion for slightly cohesive systems (McLaughlin and Rhodes, 2001; Rhodes et al., 2001; Wormsbecker and Pugsley, 2008). Very cohesive systems manifest partial, but poor, fluidization above some critical velocity where the pressure drop is considerably smaller than that required to support all the particles in the bed. As this velocity is not a good metric to characterize quality of fluidization of very cohesive systems, the notion of full-support velocity where the pressure drop is indeed commensurate with the weight of the bed has been introduced in the literature; this full-support velocity increases with degrees of wetness, or equivalently, the strength of cohesion (Wormsbecker and Pugsley, 2008; D'Amore et al., 1979). This is consistent with the increase in gas velocity required to sustain operation of a fluidized bed granulator with increased liquid loading (Maroglou and Nienow, 1985). From an operational perspective, it would be valuable to be able to predict this full-support velocity and the flow behavior in wet fluidized beds at even higher gas velocities.

There is a long history of trying to exploit simulations of fluidized suspensions to gain insights at various length scales that are difficult to extract from experiments (Deen et al., 2007; Fox, 2012; Tenneti and Subramaniam, 2014; Mohagheghi et al., 2014). Computational methods to study fluidized suspensions fall into one of three categories: (i) Direct Numerical Simulation (DNS), (ii) EulerEuler (EE), and (iii) Euler-Lagrange (EL) methods. In DNS, one solves Newton's equations of motion for the individual particles and the Navier-Stokes equations of motion for the interstitial fluid, resolving the fluid flow around the particles completely (Tenneti and Subramaniam, 2014); as such, this approach, requiring no constitutive postulates, is viable only for very small systems involving a few thousand particles, thus limiting its use to the study of micro-scale flow features. In the EE approach, both gas and solid phases are treated as interpenetrating continua and locally averaged equations (commonly referred to as two-fluid model (TFM) equations) are employed to describe the spatiotemporal structures (Gidaspow, 1994; Jackson, 2000; van der Hoef et al., 2008). This approach is, in principle, suited for the study of large scale flow characteristics, but it requires constitutive models for the fluid-particle interaction force as well as effective stresses in both phases. While considerable advances have been made in formulating the necessary constitutive models for dry systems (Gidaspow, 1994; Deen et al., 2007; Fox, 2012), the field remains primitive for wet systems. In EL methods, the fluid phase is modeled via locally averaged equations, while the particles are treated as discrete objects subject to Newton's equations of motion (Hou et al., 2012; Galvin and Benyahia, 2014). Constitutive models for the fluid-particle interaction force are needed for this approach, and it is uniformly assumed by all researchers that, at the scale of individual particles, the constitutive model for dry systems apply to wet systems as well. Deen et al. (2007) provide a review of hard and soft-sphere approaches to model particleparticle interactions, which applies to both EL and DNS methods. The Discrete Element Method (DEM) of Cundall and Strack (1979) is the most commonly used soft-sphere approach. Although EL simulations with several million particles can be handled today, industrial scale devices with billions of particles cannot be simulated unless one introduces some form of coarse-graining that cuts down the number of representative particles to be simulated (which introduces need for new constitutive models which are not yet well understood). Nevertheless, EL approaches are attractive to study meso-scale dynamics, requiring no more than a few million particles, and results from such studies can aid formulation of coarser EE and EL models (Capecelatro et al., 2014). Treating particles as discrete objects is appealing for the study of meso-scale structures in wet systems as the formation and rupture of liquid bridges between particles can easily be incorporated in the models. The present study is concerned with EL simulations of mesoscale flow characteristics in wet systems.

An introduction to the effects of liquid in granular material can be found in Herminghaus (2005). When wetted particles collide, a pendular liquid bridge is formed, giving rise to particle-particle interaction forces arising from surface tension (Mikami et al., 1998; Willett et al., 2000) and viscous (Adams and Perchard, 1985; Pitois et al., 2000; Kantak et al., 2009) effects, which depend on the physical properties of the liquid and shape of the bridge (Darabi et al., 2010). The relative importance of surface tension, viscous, and gravitational forces can be captured by a granular Bond number, $B o=\frac{6 \gamma}{d_{p}^{2} \rho_{p} g}$ and a Capillary number, $C a=\frac{\mu_{1} v_{t}}{\gamma}$. Here, $\gamma$ is the surface tension of the liquid, $d_{p}$ is the diameter of the particle, $\rho_{p}$ is the density of the particle, $g$ is the magnitude of the acceleration due to gravity, $\mu_{l}$ is the viscosity of the liquid, and $v_{t}$ is the terminal settling velocity of the particle. When particles bound by a liquid bridge are separated beyond a critical distance (Lian et al., 1993; Pitois et al., 2001), the bridge ruptures and liquid is redistributed between the particles (Shi and McCarthy, 2008). Mikami et al. (1998) show how liquid bridge models may be used in 2D simulations of wet fluidized beds, and similar models have shown success in a range of applications including mixers (Radl et al., 2010), drums (Liu et al., 2013), and hoppers (Anand et al., 2009, 2010).

Simulation of device scale systems on practical time scales requires coarsening (Agrawal et al., 2001). Coarsening a Lagrangian phase may be done by using parcels which serve as proxies for a number of other particles not directly simulated. This method, known as Multi-Phase Particle-In-Cell (MP-PIC), utilizes either collisional tracking or stress models to account for particle-particle interactions and is applicable to much larger systems (Snider, 2001; Radl and Sundaresan, 2014). Coarsening of the fluid phase is often referred to as filtering, replacing continuum averaged equations of motion with their filtered counterparts (Igci et al., 2008, 2011; Ozel et al., 2013). The purpose of coarse-graining techniques is to allow for simulation of industrial scale systems with acceptable accuracy using practical amounts of computational resources and time. Of greatest concern is the accurate prediction of the drag force, as it is the primary method by which particles are suspended in the bed. Micro-scale drag laws, such as those from Wen and Yu (1966) or Beetstra et al. (2007) assume a homogeneously distributed solids phase, an assumption that fails in coarse-grid simulations. Instabilities associated with fluidization allow for particle clustering in the system. These inhomogeneities result in a greater slip velocity being required for fluidization.

Current literature provides details regarding the effects of a liquid presence between small numbers of colliding particles 
(Pitois et al., 2000; Darabi et al., 2009; Donahue et al., 2010), yet literature describing how these microscale effects influence the dynamics of meso-scale structures and macroscale characteristics, such as secondary flows and instabilities in fluidized beds, is lacking. The work presented in this paper examines how fluidization behavior in wet gas-fluidized beds is affected by liquid bridge characteristics. However, unlike previous computational work (Rhodes et al., 2001; Darabi et al., 2011; Fries et al., 2013) that concentrated on comparably large particles in dense flows, our work focusses on gas-particle systems typical for turbulent or circulating fluidized beds. Agglomeration creates inhomogeneity within the bed, altering fluidization characteristics. For example, the gas velocity required to fluidize a collection of wet particles is larger than that required for the corresponding dry system; equivalently, the effective gas-particle drag coefficient (in TFM) is smaller for the wet system than the dry system. Flow behavior is quantitatively assessed using a domain-averaged slip velocity, which is found to be a function of particle volume fraction $(\phi)$, Bond number, and liquid loading level $(\Lambda)$, where $\Lambda$ is equal to the ratio of liquid bridge volume to particle volume. The properly scaled slip velocity varies nearly linearly with $B 0^{0.5} Z$ where $Z$ denotes the average liquid bridge coordination number, which in turn is found to be a function of $\phi^{2} B 0^{2.5} \Lambda$. A filtered drag coefficient for wet systems is found to be a function of the wetting properties, particle volume fraction, and filter size. It is shown that this wet filtered drag coefficient approaches a limit as filter size increases and that this wet filtered drag coefficient may be approximated by applying a correction to the dry filtered drag coefficient.

Note that in the current study we use small liquid loadings, i.e., less than $1 \%$ of the particle volume. Hence, we neglect the effect of the liquid on the particle mass or volume. Thus, the observed drop in the drag coefficient due to the addition of liquid is purely due to the affect on the inhomogeneity that forms in the system.

\section{Computational procedure}

\subsection{Simulation methodology}

Euler-Lagrange simulations of uniformly sized particles fluidized by a gas have been performed in 3-D periodic domains. The domain takes the form of a vertically oriented rectangular prism, with a square base and aspect ratio of 4 . Contact interaction between particles (treated as soft spheres) are represented through a linear spring dashpot model, supplemented with a frictional slider. Rolling friction is not taken into account in the present series of simulations. The discretized form of the locally averaged equations of motion describing the fluid phase are solved using cubic grids. This approach is often referred to in the literature as the CFD-DEM, Computational Fluid Dynamics-Discrete Element Method (Luding, 2008).

The boundary conditions are such that the domain is periodic in all 3 directions. That is: (1) the distance between any two points in a given direction (of domain length $\Delta_{y}$ ) is $\Delta_{y}-\left|y_{2}-y_{1}\right|$ for cases in which $\left|y_{2}-y_{1}\right|>\Delta_{y} / 2$, and (2) particles leaving through one side of the domain enter in the opposing side with identical velocities.

When the particles are wet, collision between two particles will lead to the formation of a liquid bridge. The rate of filling of the liquid bridge will be affected by liquid viscosity (Mohagheghi et al., 2014), but we assume in this study, for the sake of simplicity, that the liquid bridge fills instantaneously. This assumption is valid as long as the typical particle-particle interaction time is larger than the characteristic time scale due to bridge filling. The latter is $d_{p} \mu_{l} / \gamma_{l}$, and for the systems considered in our study is on the order of $2 \mu \mathrm{s}$ when considering the surface tension and viscosity of water. Thus, filling occurs much faster than the re-arrangement of the particles in our system, which occurs within a typical time scale of $50 \mathrm{~ms}$ (i.e., the relaxation time of the particles, $v_{t} / g$ ).

Strictly speaking, when a liquid-coated particle surrenders some liquid to the bridge, it has less free liquid available to form new bridges with other particles with which it may collide. In this study, we avoid this complexity and postulate that when two particles collide, a liquid bridge containing a fixed amount of liquid (which is user-specified and treated as a parameter characterizing the degree of wetness) forms; this bridge results in an attractive surface tension interaction force between the particles (Mikami et al., 1998; Willett et al., 2000), which is described in greater detail below. Relative motion between the particles can also cause a viscous force due to the liquid bridge (Adams and Perchard, 1985; Pitois et al., 2000; Kantak et al., 2009), but we do not consider this force in the present study, which is aimed at shedding qualitative insight into the flow characteristics that can be attributed to the surface tension force in the simplest possible manner. We present in Appendix A the results obtained in simulations where liquid transfer between particles is considered. Such simulations follow the fluidization dynamics of a collection of uniformly wetted particles with a specified total amount of liquid in the domain. Liquid bridges will contain different amounts of liquids in such cases, but, as discussed in Appendix A, the outcome is similar to what we obtain for the simpler case of fixed liquid bridge volume considered here.

Although all results will be presented in terms of dimensionless quantities, we provide in Table 1 values of various dimensional, physical, and computational parameters for a typical physical system.

Particle motion is followed by solving Newton's equations of motion:

$m_{i} \frac{d \mathbf{v}_{i}}{d t}=\sum_{j}\left(\mathbf{f}_{c o n t, i j}^{n}+\mathbf{f}_{c o n t, i j}^{t}\right)+\sum_{k} \mathbf{f}_{b, i k}+\mathbf{f}_{g \rightarrow p, i}+m_{i} \mathbf{g}$

$I_{i} \frac{d \omega_{i}}{d t}=\boldsymbol{q}_{i}$

Here, particle $i$ has mass $m_{i}$ and translational velocity $\mathbf{v}_{i} . \mathbf{f}_{\text {cont }, i j}^{n}$ is the normal contact force acting on particle $i$ by particle $j, \mathbf{f}_{c o n t, i j}^{t}$ is

Table 1

Typical values of dimensional parameters used in the simulations.

\begin{tabular}{ll}
\hline Parameter & Value \\
\hline Domain size $-x ; \Delta_{x}(\mathrm{~m})$ & 0.008 \\
Domain size $-y ; \Delta_{y}(\mathrm{~m})$ & 0.032 \\
Domain size $-z ; \Delta_{z}(\mathrm{~m})$ & 0.008 \\
Number of fluid grids $-x ; N_{x}$ & 18 \\
Number of fluid grids $-y ; N_{y}$ & 72 \\
Number of fluid grids $-z ; N_{z}$ & 18 \\
Acceleration due to gravity; $\left(\mathrm{m} / \mathrm{s}^{2}\right)$ & 9.81 \\
Particle diameter; $d_{p}(\mathrm{~m})$ & $1.45 \times 10^{-4}$ \\
Particle density; $\rho_{p}\left(\mathrm{~kg} / \mathrm{m}^{3}\right)$ & 1600 \\
Normal spring constant; $k_{n}\left(\mathrm{~kg} / \mathrm{s}^{2}\right)$ & 2.05 \\
Normal spring damping; $\gamma_{d, n}(\mathrm{~kg} / \mathrm{s})$ & $3.43 \times 10^{-6}$ \\
Tangential spring constant; $k_{t}\left(\mathrm{~kg} / \mathrm{s}^{2}\right)$ & 0.586 \\
Tangential spring damping; $\gamma_{d, t}(\mathrm{~kg} / \mathrm{s})$ & $3.43 \times 10^{-6}$ \\
Friction coefficient; $\mu_{p p}$ & 0.5 \\
Restitution coefficient of the dry particles; $e_{p p}$ & 0.9 \\
Gas density; $\rho_{g}\left(\mathrm{~kg} / \mathrm{m}^{3}\right)$ & 2.28 \\
Gas viscosity; $\mu_{g}(\mathrm{~Pa} \mathrm{~s})$ & $2.5 \times 10^{-5}$ \\
Particle terminal settling velocity; $v_{t}(\mathrm{~m} / \mathrm{s})$ & 0.5 \\
Froude number; $F r=v_{t}^{2} /\left(d_{p} g\right)$ & 180 \\
Particle timestep; $\Delta_{t p}(\mathrm{~s})$ & $10^{-6}$ \\
Fluid timestep; $\Delta_{t g}(\mathrm{~s})$ & $10^{-5}$ \\
\hline
\end{tabular}


the tangential contact force acting on particle $i$ by particle $j, \mathbf{f}_{b, i k}$ is the liquid bridge force acting on particle $i$ by bridge $k, \mathbf{f}_{g \rightarrow p, i}$ is the interaction force on the particles due to fluidizing gas (explained further below), $I$ is the moment of inertia, $\omega$ is the angular velocity, and $\mathbf{q}$ is the total torque.

Particle-particle interactions consist of a standard springdashpot model and frictional slider (Cundall and Strack, 1979):

$\mathbf{f}_{c o n t, i j}^{n}=-k_{n} \delta_{i j} \mathbf{n}_{i j}-\gamma_{d, n} \mathbf{v}_{i j}^{n}$

$\mathbf{f}_{\text {cont }, i j}^{t}= \begin{cases}-k_{t} \mathbf{t}_{i j}-\gamma_{d, t} \mathbf{v}_{i j}^{t} & \text { for }\left|\mathbf{f}_{c o n t, i j}^{t}\right|<\mu_{p p}\left|\mathbf{f}_{c o n t, i j}^{n}\right| \\ -\mu_{p p}\left|\mathbf{f}_{c o n t, i j}^{n}\right| \frac{\mathbf{t}_{i j}}{\left|\mathbf{t}_{i j}\right|} & \text { for }\left|\mathbf{f}_{\text {cont }, i j}^{t}\right| \geq \mu_{p p}\left|\mathbf{f}_{c o n t, i j}^{n}\right|\end{cases}$

Here, $k_{n}$ is the normal spring constant, $\delta_{i j}$ is the normal overlap between particles $i$ and $j, \mathbf{n}_{i j}$ is the unit normal vector pointing from particle $i$ to collision partner $j, \gamma_{d, n}$ is the normal damping coefficient, $\mathbf{v}_{i j}^{n}$ is the relative normal velocity of particles $i$ and $j, k_{t}$ is the tangential spring constant, $\mathbf{t}_{i j}$ is the tangential overlap obtained from the integration of the relative tangential velocity between contacting particles, $\gamma_{d, t}$ is the tangential damping coefficient, $\mathbf{v}_{i j}^{t}$ is the relative tangential velocity of particles $i$ and $j$, and $\mu_{p p}$ is the friction coefficient. We note that parameters of the spring-dashpot model (shown in Table 1) are chosen such that the restitution coefficient of the dry particles $\left(e_{p p}\right)$ is 0.9 . In order to decrease computational cost, simulations typically treat particles as softer than they really are. It is important to ensure that the flow structures and quantities extracted from the simulations are not dependent on the degree of softness. With this in mind, simulations were done with different spring constants and time steps to ensure that results were not sensitive to changes in these parameters. The time step (shown in Table 1) was chosen to be between $1 / 50$ and $1 / 100$ of the binary dry collision time for all data presented here. The fluid grid size was chosen to be approximately $3 d_{p}$ as suggested by Radl and Sundaresan (2014), yielding grid independent results. Results confirming that the grid size is sufficiently small are shown in Appendix B.

To model the effect of liquid bridges, two approaches exist: (i) using a "wet" restitution coefficient (Antonyuk et al., 2009; Sutkar et al., 2015), or (ii) using force models that directly predict the liquid bridge force. Approach (i) is only approximate, since it cannot model enduring cohesive contact forces. Also, there is still significant uncertainty connected in modeling the "wet" restitution coefficient (Sutkar et al., 2015). Approach (ii) is more universal, however, one must choose from a plethora of liquid bridge force models (Antonyuk et al., 2009). The latter introduces a significant amount of dimensionless parameters that need to be considered (e.g, a scaled particle roughness, or a particle Stokes number to quantify the effect of the liquid viscosity). Here we follow the work of Mikami et al. (1998) in modeling the liquid bridges between particles, which only accounts for capillary forces. This approach is valid for low particle-particle relative velocities and rough particles, for which viscous forces become insignificant. When particles come into contact, a pendular liquid bridge of set volume is immediately formed. This liquid bridge provides a cohesive force of the form:

$$
\begin{aligned}
& \mathbf{f}_{b, i k}=\mathbf{n}_{i b} \pi r \gamma\left(\mathrm{e}^{A \hat{h}+B}+C\right) \\
& A=-1.1 \hat{V}^{-0.53} \\
& B=(-0.34 \ln \hat{V}-0.96) \theta^{2}-0.019 \ln \hat{V}+0.48 \\
& C=0.0042 \ln \hat{V}+0.078
\end{aligned}
$$

Here, $\mathbf{n}_{i b}$ is the unit normal vector pointing from particle $i$ to bridge $b, \hat{h}$ is the surface to surface separation distance scaled by $r$ where $r$ denotes the particle radius, $\hat{V}$ is the liquid bridge volume made dimensionless by scaling with $r^{3}(\hat{V}=4 \pi \Lambda / 3)$, and $\theta$ is the contact angle which is assumed to be zero. The cohesive force is finite at particle-particle overlap and is taken to be a constant equal to the cohesive force at zero surface-surface separation distance. When a liquid bridge reaches a critical distance, the bridge ruptures and liquid is redistributed. This critical liquid bridge rupture distance is given by Lian et al. (1993):

$\hat{h}_{c}=(1+0.5 \theta) \hat{V}^{1 / 3}$

The liquid bridge force is present as long as a bridge exists. Particles are allowed to form multiple pairwise interactions via liquid bridges and in this way agglomerates form. The constant liquid bridge model used in this work is compared with a more advanced liquid transfer model in Appendix A.

The fluid phase is modeled as a continuum and its motion is governed by conservation of mass and momentum (Zhou et al., 2010):

$\frac{\partial}{\partial t}[(1-\phi)]+\nabla \cdot\left[(1-\phi) \mathbf{u}_{g}\right]=0$

$\rho_{g}(1-\phi)\left(\frac{\partial \mathbf{u}_{g}}{\partial t}+\mathbf{u}_{g} \cdot \nabla \mathbf{u}_{g}\right)=-\nabla p_{g}+\nabla \cdot \boldsymbol{\tau}_{g}+\boldsymbol{\Phi}_{d}+\rho_{g}(1-\phi) \mathbf{g}$

Here, $\rho_{g}$ is the density of the gas, $\phi$ is the solid volume fraction, $\mathbf{u}_{g}$ is the gas velocity, $p_{g}$ is the gas phase pressure, and $\boldsymbol{\tau}_{g}$ is the gas phase deviatoric stress tensor. The total gas-particle interaction force per unit volume of the mixture, exerted on the particles by the gas, $-\boldsymbol{\Phi}_{d}$, is composed of a generalized buoyancy force due to the slowly varying (in space) local-average gas phase stress $\left(-p_{g}\right.$ $\mathbf{I}+\boldsymbol{\tau}_{g}$ ) and the force due to the rapidly varying flow (in space) field around the particles. On a per particle basis, the total interaction force on the particle by the gas can be written as $\mathbf{f}_{g \rightarrow p, i}=-$ $V_{p, i} \nabla p_{g, i}+V_{p, i} \nabla \cdot \boldsymbol{\tau}_{g, i}+\mathbf{f}_{g \rightarrow p, i}^{\prime}$, where $V_{p, i}$ is the particle volume and $\mathbf{f}_{g \rightarrow p, i}^{\prime}$ is the gas-particle force due to fluid flow around the particle. Subscript $i$ indicates that quantities are per particle, and that fluid phase properties have been interpolated at the particle position. In this study, we only consider the drag force, denoted as $\mathbf{f}_{d, i}$, which is the most important component of $\mathbf{f}_{g \rightarrow p, i}^{\prime}$ for gas-particle flows (as the particle density is much greater than the gas density). For other forces see Sommerfeld et al. In gas-fluidized beds of particles, $\boldsymbol{\tau}_{g}$ does not play a significant role, so discarding this term makes little difference. For $\mathbf{f}_{d, i}$, we select the drag model proposed by Beetstra et al. (2007) as shown in Eq. (12). Here, $R e_{p}$, is the particle-Reynolds number (Eq. (14)), $d_{p}$ is the particle diameter, $\mathbf{v}_{p}$ is the velocity of the particle, and $\mu_{g}$ is the viscosity of the fluidizing gas phase:

$$
\begin{aligned}
\mathbf{f}_{d, i}= & 3 \pi \mu_{g} d_{p}(1-\phi)\left(\mathbf{u}_{g}-\mathbf{v}_{p}\right) \hat{f}_{d, i} \\
\hat{f}_{d, i}= & \frac{10 \phi}{(1-\phi)^{2}}+(1-\phi)^{2}\left(1+1.5 \phi^{1 / 2}\right) \\
& +\frac{0.413 R e_{p}}{24(1-\phi)^{2}}\left[\frac{(1-\phi)^{-1}+3 \phi(1-\phi)+8.4 R e_{p}^{-0.343}}{1+10^{3 \phi} \operatorname{Re}_{p}^{-(1+4 \phi) / 2}}\right] \\
\operatorname{Re}_{p}= & \frac{(1-\phi) \rho_{g}\left|\mathbf{u}_{g}-\mathbf{v}_{p}\right| d_{p}}{\mu_{g}}
\end{aligned}
$$

We have verified the correct implementation of the above models by considering a variety of test cases (e.g., sedimentation of a single particle, sedimentation of a homogeneous suspension, and binary collision of dry and wet particles). Unfortunately, it is not possible to validate the current predictions for sedimentation in a periodic domain, but only predictions for bounded domains (e.g., the minimum fluidization velocity). The latter has been done for the set of models used in the current study, and for dry 
particles in a number of previous studies (Vincent et al., 2014). These tests are also done by our group to ensure the quality of the simulation code used. We hence conclude that the models used in the current study yield realistic predictions, despite the fact that an experimental validation was not possible.

\subsection{Coarse-grained quantities extracted from simulations}

From the above microscale simulations, we first extract domain-average gas and particle velocities as:

$\left\langle\mathbf{u}_{g}\right\rangle=\frac{1}{V_{d}\left\langle\phi_{g}\right\rangle} \int\left(\phi_{g} \mathbf{u}_{g}\right) d V$

$\left\langle\mathbf{u}_{s}\right\rangle=\frac{1}{N_{p}} \sum^{N_{p}} \mathbf{u}_{s}$

Here \langle\rangle indicate domain-average quantities, $V_{d}$ denotes domain volume, and $N_{p}$ is the number of particles in the simulation domain.

We then seek filtered quantities (also represented by angle brackets) where the size of the filter is smaller than the domain size. In such filtering analysis, we consider filters of specified size whose center is coincident with the center of each microscale grid, with filter size typically being odd multiples of the microscale grid size. Each microscale grid acts as the center of a larger coarser grid. The side length of the coarser grid is 3-9 times the length of the microscale grid. The fluid properties recorded by the microscale fluid grids, specifically the fluid velocity $\left(\mathbf{u}_{g}\right)$, pressure gradient $\left(\nabla p_{g}\right)$, and solid volume fraction $(\phi)$, may then also be volumeaveraged over the filter to obtain the filtered quantities $\left(\left\langle\mathbf{u}_{g}\right\rangle, \nabla\left\langle p_{g}\right\rangle,\langle\phi\rangle\right)$. Such filtered quantities for different filter sizes are gathered and stored at each microscale grid.

The purpose of such filtering analysis is to formulate corrected drag laws which should be used if one wishes to perform CFDDEM simulations with coarser fluid grids, while accounting for the consequences of sub-grid scale fluid flow structures in a suitable average sense. (Using the microscopic drag law mentioned earlier with no modification is equivalent to ignoring the effects of these sub-grid structures which, as we will demonstrate later, leads to very poor predictions.) The fluid-particle interaction force in microscale simulations is given by: $\mathbf{f}_{g \rightarrow p, i}=-V_{p, i} \nabla p_{g}+\beta\left(\mathbf{u}_{g}-\mathbf{v}_{p, i}\right)$. If only filtered fluid velocity, pressure, and volume fractions fields are available, then $\mathbf{f}_{g \rightarrow p, i}=-V_{p, i} \nabla\left\langle p_{g}\right\rangle+\langle\beta\rangle\left(\left\langle\mathbf{u}_{g}\right\rangle-\mathbf{v}_{p, i}\right)$, where $\langle\beta\rangle$ is an effective drag coefficient (as of yet undetermined).

In this manner, one can determine an effective drag coefficient for each particle in the simulation domain (for each chosen filter size). These $\langle\beta\rangle$ values are then stored in various bins corresponding to different $\left\langle\phi_{s}\right\rangle$ windows; they are then averaged to produce ensemble average estimates. (In the present study, we did not consider additional markers such as filtered slip velocity (Milioli et al., 2013) to more finely bin the filtered quantities.) We also determined and binned $\langle\beta\rangle / \beta_{\text {micro }}$ where $\beta_{\text {micro }}$ was determined using the microscale drag law and the filtered fluid volume fraction and velocity. In general, $\langle\beta\rangle$ and $\langle\beta\rangle / \beta_{\text {micro }}$ depend on filter size, filtered particle volume fraction, and the liquid bridge characteristics ( $B o$ and $\Lambda$ ).

\subsection{Simulation procedure}

Transient simulations are initialized with a nearly homogeneous distribution of particles in the domain. The initial velocities of all the particles are set to zero. The initial fluid velocity in every cell is set to be that corresponding to a homogeneously fluidized state where the drag force balances the weight of each

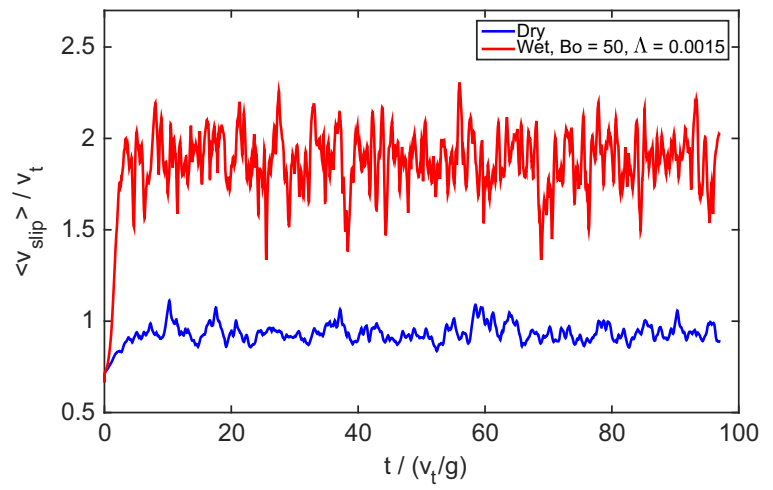

Fig. 1. Scaled domain-averaged slip velocity vs. dimensionless time. Particle volume fraction in the domain is 0.05 . See Table 1 for values of parameters used in the simulations. An initially homogeneous assembly of particles, subjected to a pressure drop that exactly balances the weight of the suspension per unit cross sectional area, develops inhomogeneous structures, which leads to the initial increase in the domain-averaged slip velocity. Eventually, the system attains a statistical steady state. The figure compares the dynamics in dry and wet systems.

particle. Inhomogeneities emerge over a period of time as a result of instabilities associated with fluidization as well as formation of liquid bridges. The evolution of inhomogeneity can be recognized in at least two ways: (a) snapshots of particle volume fraction distribution, and (b) the domain-averaged slip velocity $\left(\left\langle v_{\text {slip }}\right\rangle\right)$ required to fully support the weight of all the particles. $\left\langle v_{\text {slip }}\right\rangle$ is defined as the difference between the Favre-average velocity of the gas in the domain and the average velocity of all the particles in the domain. Fig. 1 displays $\left\langle v_{\text {slip }}\right\rangle$ as a function of time for typical dry and wet systems. In the present study we wait for every simulation to reach a statistical steady state and collect snapshots for subsequent post-processing. The domain-averaged slip velocity in the statistical steady state, which can readily be extracted from data of the type shown in Fig. 1, is an important quantitative metric in our study. We have ascertained that $\left\langle v_{\text {slip }}\right\rangle$ in the statistical steady state is independent of the initial condition employed by starting several simulations from different initial conditions. The domains studied are on the order of cubic centimeters, with the number of particles in any given simulation ranging from 64,000 to over half a million, while fluid grid number ranged between 23,000 and 79,000. Over two hundred simulations have been performed over a range of particle volume fractions, and liquid bridge volume, Bo. The results from these simulations are discussed in the remaining sections.

\section{Results and discussion}

\subsection{Influence of the wetting properties on flow behavior}

As seen in Fig. $1,\left\langle v_{\text {slip }}\right\rangle$ in the statistical steady state is, in general, larger than that for a homogeneously fluidized state even for dry systems, which can be traced to the emergence of inhomogeneous structures (clusters in dilute suspensions and bubble-like voids in denser suspensions). However, $\left\langle v_{\text {slip }}\right\rangle$ in wet systems is even larger than those for the corresponding dry cases (see Fig. 1), and it can be readily attributed to the liquid-bridge induced agglomeration of particles. To illustrate this, we present, in Fig. 2, snapshots for a dry system and several wet systems covering a range of Bond numbers. Inhomogeneity in the form of clusters, that is observed in dry systems (snapshot (a) in Fig. 2) is typically attributed to inelastic collisions and instability associated with the uniformly fluidized state (Glasser et al., 1998; Li and Kuipers, 2005; van der Hoef et al., 2006, 2008). Aggregates resulting from cohesive forces due to the liquid bridges (snapshots (b)-(e) in Fig. 2) 

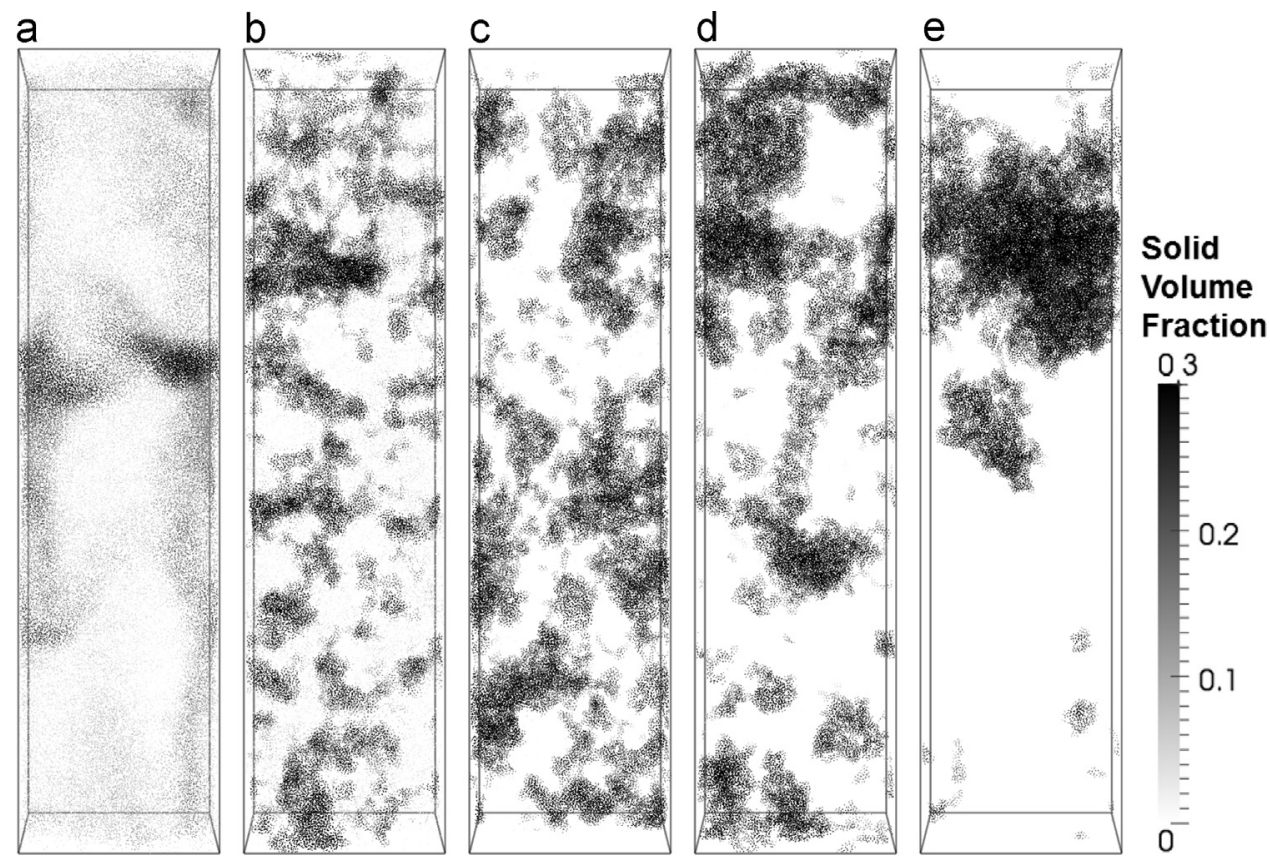

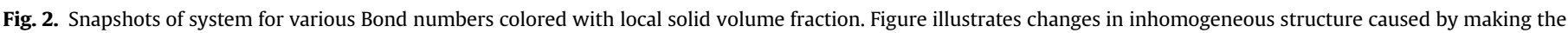

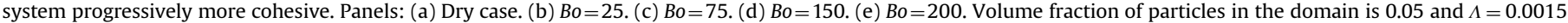
for wet cases. Values of other parameters used in the simulations are listed in Table 1.
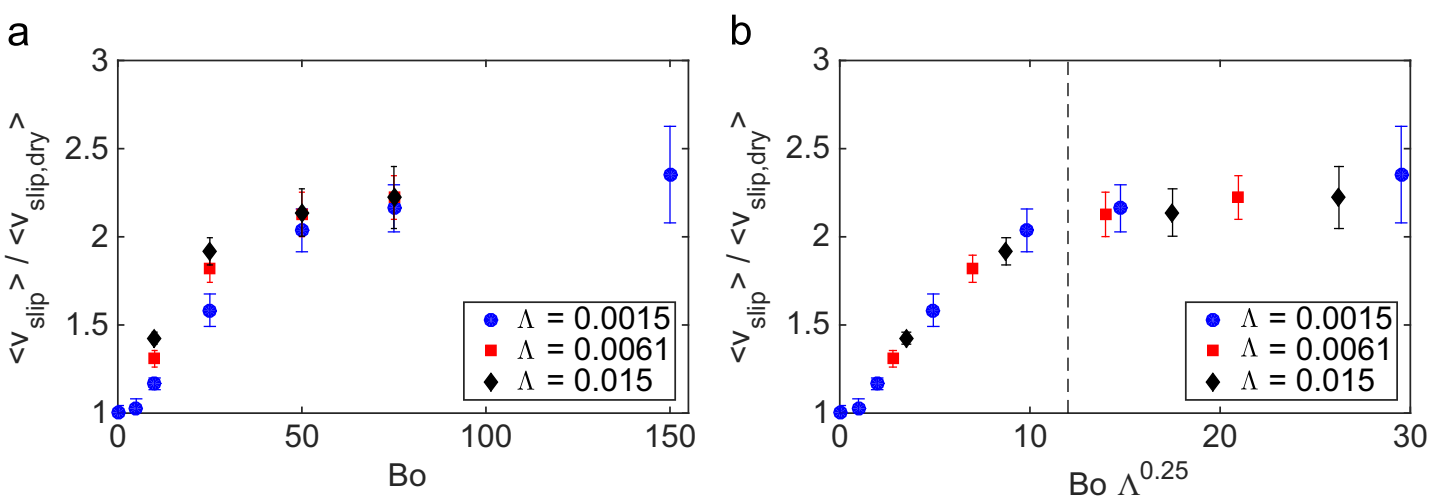

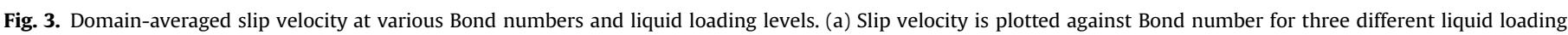

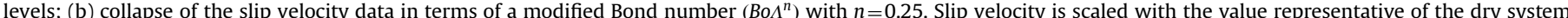

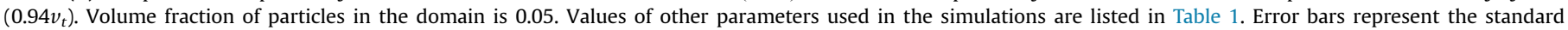
deviation in the domain-averaged slip velocity as measured over time.

are clearly very different from and more extensive than those occurring in a dry system. It is clear from Fig. 2 that as the particles become more cohesive, the agglomerates become larger; this, in turn, requires larger $\left\langle v_{\text {slip }}\right\rangle$ to fluidize the particles.

It is important to note that the pressure drop across the height of the simulation domain is maintained unaltered throughout the simulation. The formation of agglomerates tends to lower fluidparticle interaction, as the agglomerates present themselves as fewer larger particles. As a result, the effective fluid-particle drag coefficient decreases and a greater slip velocity is needed to achieve the same pressure drop.

We present in Fig. 3a the domain-averaged slip velocities extracted from statistical steady states in simulations performed at various $B o$ and $\Lambda$ values. As the liquid bridges are made more cohesive, either by increasing Bo or $\Lambda$, the extent of inhomogeneity in the domain increases, resulting in an increase in $\left\langle v_{\text {slip }}\right\rangle$. The results in Fig. 3a can be collapsed reasonably well into a single master curve by defining a modified Bond number, $B o \Lambda^{n}$, where $n=0.25 \pm 0.05$. $\left\langle v_{\text {slip }}\right\rangle$ as a function of the modified Bond number, with $n=0.25$ is shown in Fig. $3 \mathrm{~b}$. The principal message of Fig. $3 \mathrm{~b}$ is that one should be able to rationalize the effects of $B o$ and $\Lambda$ in a common framework (which will be discussed below). The error bars in Fig. 3 represent the standard deviation in the domainaveraged slip velocity as measured over time; see temporal fluctuations earlier in Fig. 1. The more cohesive the particles are, the larger the fluctuations are.

While it is difficult to directly compare the results of our study with experimental data, we note that Zhou et al. (2012) see a similar trend (i.e., increasing pressure fluctuations with increasing liquid content). Unfortunately, a more quantitative comparison of the effect of liquid content on the fluidization behavior is not possible. This is because the experimental measurement of temporal fluctuations of the slip velocity in dilute fluidized beds is currently not possible.

For sufficiently large values of Bond number and/or liquid loading level, simulation yields just one agglomerate that spans the width of the domain (as shown in Fig. 2e). Proper fluidization is not achieved when this happens, and meaningful statistics can 

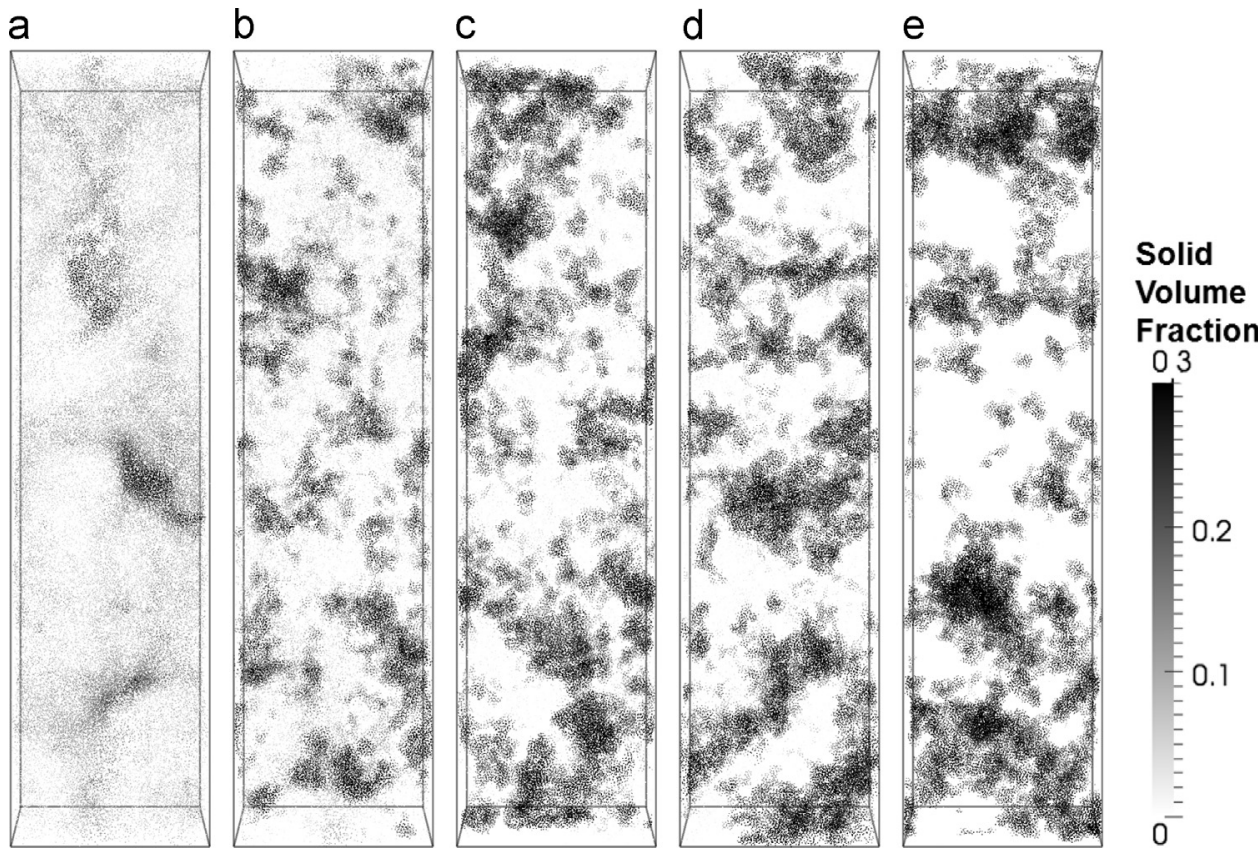

Fig. 4. Snapshots of wet systems taken for various Bond numbers and liquid loading levels colored with local solid volume fraction. Panels: (a) $B 0=5, \Lambda=0.0015, B 0 \Lambda^{0.25}=0.98$. (b) $B o=10, \Lambda=0.0061, B o \Lambda^{0.25}=2.8$. (c) $B o=25, \Lambda=0.0061, B o \Lambda^{0.25}=7.0$. (d) $B o=50, \Lambda=0.0015, B o \Lambda^{0.25}=9.8$. (e) $B o=75, \Lambda=0.015$, Bo $\Lambda^{0.25}=26$. Volume fraction of particles in the domain is 0.05 . Values of other parameters used in the simulations are listed in Table 1.
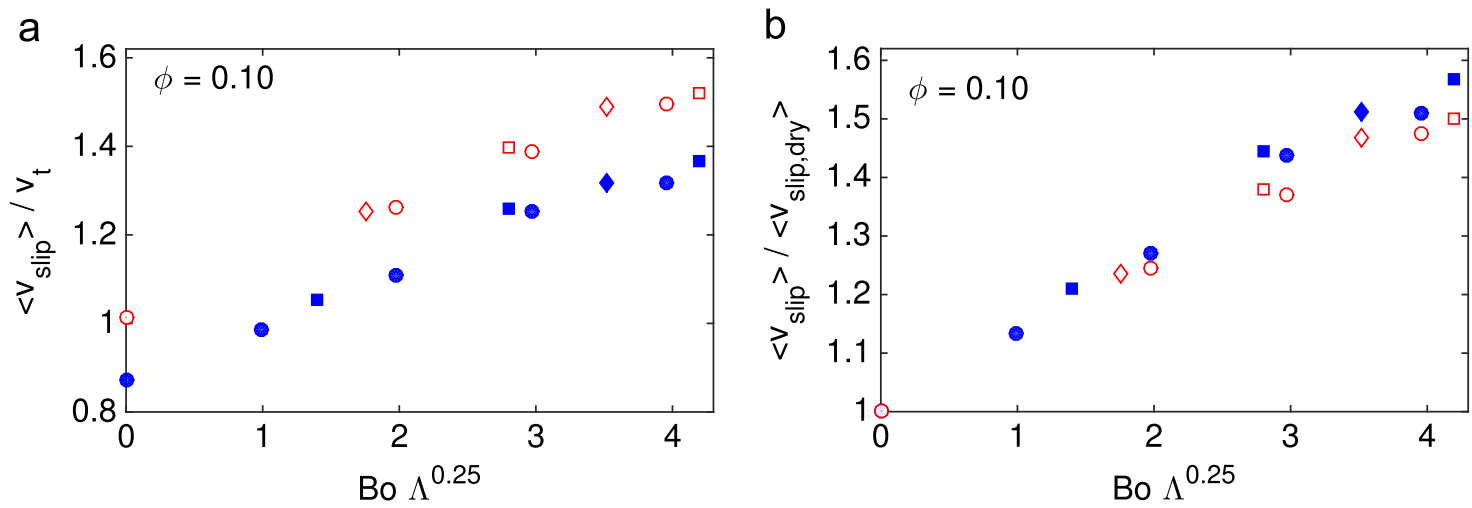

Fig. 5. Domain-averaged slip velocity vs. modified Bond number for two different domain sizes. Filled symbols represent the default domain (as in Table 1 , with $18 \times 72 \times 18$ fluid grids) and empty symbols represent a larger domain that is 1.5 times longer in each direction (with $27 \times 108 \times 27$ fluid grids). The fluid grid size is the same for both domains $\left(3.07 d_{p}\right)$. Solid volume fraction in the system is 0.10 . $\circ: \Lambda=0.0015, \square: \Lambda=0.0061, \diamond: \Lambda=0.015$. Slip velocity in the dry system is $0.87 v_{t}$ and $1.01 v_{t}$ for the default and large domain respectively. Values of other parameters used in the simulations are listed in Table 1.

no longer be collected. One can readily conclude that this outcome is a limitation caused by the small domain employed in our simulations, which can, in principle, be removed by performing simulations in much larger domains; however, the computational cost is prohibitive and beyond our resources. Strictly speaking, the statistics gathered in simulations of the type performed in our study are meaningful only when the agglomerates are considerably smaller than the domain size and there are many agglomerates in the domain. Thus, for example, the results obtained from the simulation leading to snapshot (e) in Fig. 2 are not representative of what one is likely to see in real systems. Although we illustrate the formation of a single large agglomerate in Fig. 2e, we have not included such unphysical states in the results presented in Fig. 3. Thus, Fig. 3 displays results for cases where several agglomerates could be seen in the snapshots. We note that for the results in Fig. 3 to be representative of real wet fluidized beds, the agglomerate size must be smaller than the domain width.
Fig. 4 shows snapshots for wet systems corresponding to various abscissa values in Fig. 3b: panel (a) is obtained for a condition corresponding to very small abscissa values; panels (b) and (c) are from conditions in the steeply rising region in Fig. $3 \mathrm{~b}$; (d) is at the transition from the steeply rising region to the plateau region, and (e) is in the plateau region. (We analyzed many such snapshots gathered at different times in the statistical steady state, but only one snapshot is presented per case.) Panels (d) and (e) reveal agglomerates with sizes comparable to the width of the domain, but the other panels show the presence of comparatively smaller agglomerates. Fig. 4 suggests that the results falling in the steeply rising portion of Fig. 3 can be taken as representative of real physical systems and that the emergence of the plateau region may be a limitation imposed by the domain size used in the simulations. We hypothesize that $\left\langle v_{\text {slip }}\right\rangle$ would continue to increase with $B o$ (in Fig. 3a) without plateauing in real fluidized beds, which are not subject to the small periodic domain restriction of our simulations. We performed simulations in a modestly larger domain by increasing the domain size by a factor of 1.5 in each 
a

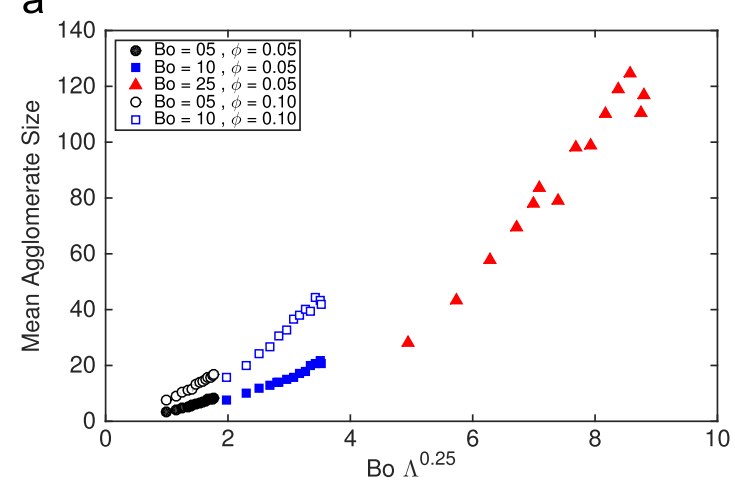

b

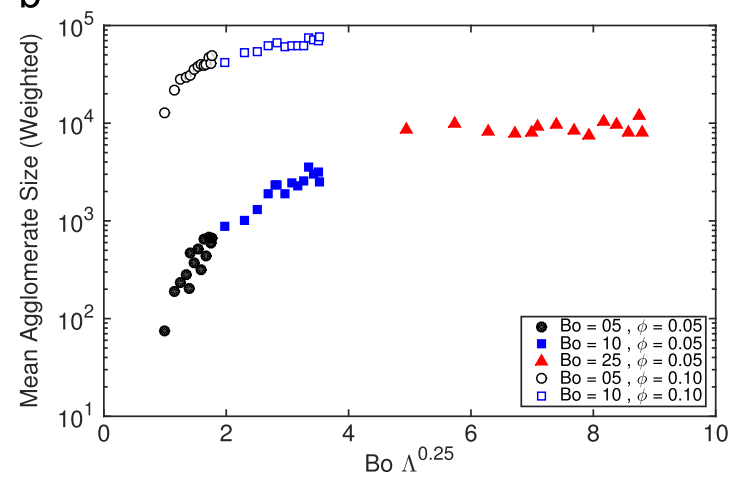

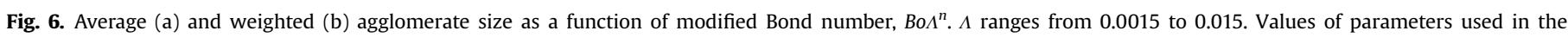
simulations are listed in Table 1.

direction, and compared the results with the base case in Fig. 5a and $\mathrm{b}$ for $\phi=0.10$. In general, $\left\langle v_{\text {slip }}\right\rangle$ increases with domain size for both wet and dry systems, but $\left\langle v_{\text {slip }}\right\rangle$ in wet systems, scaled by $\left\langle v_{\text {slip }}\right\rangle$ for corresponding dry systems (see caption for Fig. 5), does not show any reliably consistent dependence on domain size. The differences between the filled and open symbols in these figures are no greater than the fluctuations in the slip velocities in the statistical steady state (indicated earlier in Fig. 3). The transition to the plateau region occurs at nearly the same abscissa value for both smaller and larger domains; we suspect that this is due to the rapid growth in agglomerate size near the transition point, rendering modest increase in domain size insufficient to see the domain size effect.

Two observations can be made by comparing Figs. 3b and 5b. First, the idea of a modified Bond number, which was introduced in Fig. 3b for $\phi=0.05$, persists at $\phi=0.10$ as well. Second, the transition from the steeply rising region to the plateau region appears at smaller abscissa values when $\phi$ is increased, which is consistent with the agglomerate size based argument, as larger agglomerates form more easily at higher $\phi$ values. This is consistent with our hypothesis that the steeply rising region would extend to much larger (modified) Bond numbers for very large domains, but does by no means prove it. Henceforth, we focus our discussion on the steeply rising region only, indicated by the region left of the dashed line in Fig. $3 \mathrm{~b}$.

Agglomerate sizes may be obtained through the liquid bridge networks reported from the simulations. We define an agglomerate as any set of particles connected by a network of liquid bridges. Fig. 6a shows the mean agglomerate sizes for various Bond numbers and liquid loading levels. Particles not involved in any liquid bridges are excluded in this analysis and the mean agglomerate size is defined as the total number of particles involved in agglomerates divided by the total number of agglomerates: $\bar{n}=\frac{1}{m} \sum_{i=1}^{m} n_{i}$. Here $\bar{n}$ denotes the mean agglomerate size, $n_{i}$ the number of particles in agglomerate $i$, and $m$ is the total number of agglomerates. The average is computed for 15 snapshots in the statistically steady state region, with each snapshot spaced at an interval of one characteristic time step $\left(v_{t} / g\right)$. There are 64,150 particles in the 0.05 solid volume fraction system, and 128,300 particles in the 0.10 solid volume fraction system. It is seen from Fig. 6 a that the mean agglomerate size can also be thought of as a function of a function of $B o \Lambda^{n}$. Fig. $6 \mathrm{~b}$ presents the mean weighted agglomerate size, $\bar{n}_{w}=\sum_{i=1}^{m} n_{i}^{2} / \sum_{i=1}^{m} n_{i}$. It is clear that $\bar{n}_{w}$ increases with $B o \Lambda^{n}$ as well.

Besides Bond number and liquid loading level, the present problem involves two additional particle-scale dimensionless groups: Reynolds number $\left(R e_{p}=\rho_{g} d_{p} v_{t} / \mu_{g}\right)$ and Froude number $\left(F r_{p}=v_{t}^{2} /\left(d_{p} g\right)\right)$. For the base case discussed thus far, $R e_{p}=6.7$ and $F r_{p}=180$. We found that, for the case of dry particles, varying $\operatorname{Re}_{p}$

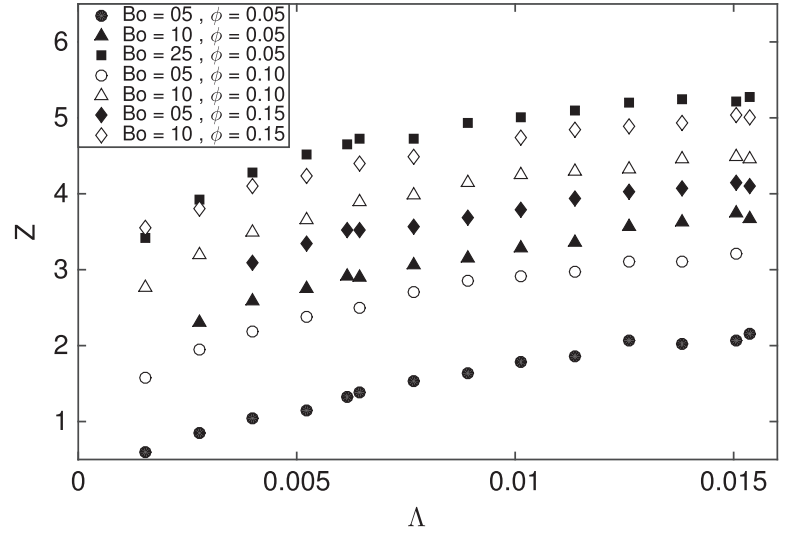

Fig. 7. Average liquid bridge coordination number $(Z)$ vs. liquid loading level $(\Lambda)$ for various $B o$ and solid volume fractions $(\phi)$. Values of other parameters used in the simulations are listed in Table 1.

between 3.4 and 20 showed $<5 \%$ change in $\left\langle v_{\text {slip }}\right\rangle$, while varying $\mathrm{Fr}_{p}$ between 15 and 360 showed $<15 \%$ change in $\left\langle v_{\text {slip }}\right\rangle$. In comparison, adding a moderate amount of liquid $(\Lambda=0.0015, B o=25)$ increases $\left\langle v_{\text {slip }}\right\rangle$ by nearly $60 \%$. Thus, although $F r_{p}$ and to a lesser extent, $R e_{p}$ do affect the fluidization behavior, the impact of wetness is much greater. In view of this observation and the expensive nature of the simulations, we have focused on studying the effects of $B o$ and $\Lambda$, while holding $F r_{p}$ and $R e_{p}$ constant.

The modified Bond number, $B o \Lambda^{n}$, was introduced earlier to simply point out that there is a rational way of placing the effect of cohesion in a simple framework. To probe this framework further, we determined the average liquid bridge coordination number $(Z)$, which is the average number of liquid bridges per particle in the domain. Fig. 7 shows the variation of $Z$ with $\Lambda$ for various $B o$ and $\phi$. As one would intuitively expect, $Z$ increases with $\Lambda$; and, at fixed $\Lambda, Z$ increases with $B o$ as well as $\phi$. We found that the scaled slip velocity for several different $B o, \phi$, and $\Lambda$ values increases nearly linearly with $B o^{0.5} Z$ as shown in Fig. 8 .

One limitation in using Fig. 8 is that $Z$ is not an input variable. Hence, to be of practical value, one must also formulate a correlation expressing $Z$ in terms of input quantities. We found empirically that all the results in Fig. 7 as well as those from many other simulations can be collapsed into a single master curve, as shown in Fig. 9. This allows us to express $Z$ as a function of $\phi^{2}$ $B o^{2.5} \Lambda$ (which is an input to the simulations). In arriving at the expression indicated in Fig. 9, we set the value of $Z$ for large abscissa values to be 6 , which was approximately the average $Z$ in simulations leading to single large agglomerates (as in Fig. 2e). We then regressed the other constants in the indicated expression. The results in Fig. 10 are the same as those in Fig. 8, except that $Z$ is 


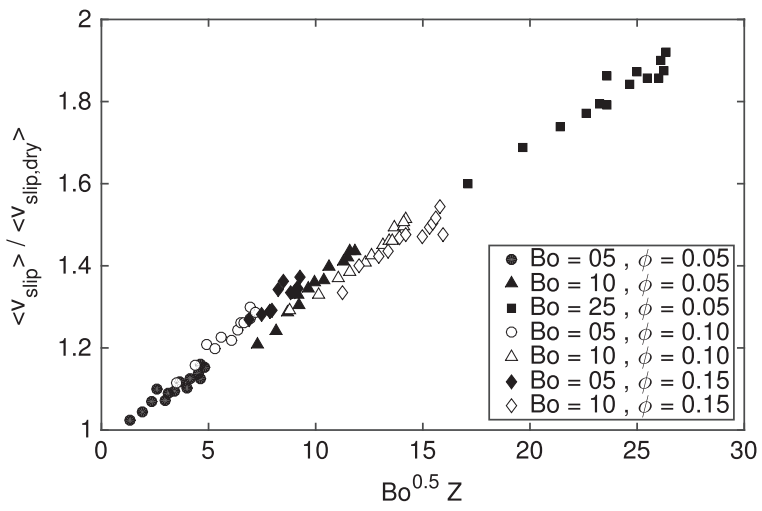

Fig. 8. Domain-averaged slip velocity vs. product of $B o^{0.5}$ and average liquid bridge coordination number $(Z)$. The figure contains results obtained in simulations at different domain-averaged particle volume fractions, Bond numbers, and liquid loading levels. Slip velocity is scaled with the value representative of the dry system $\left(0.94 v_{t}, 0.87 v_{t}\right.$, and $0.78 v_{t}$ for particle volume fractions of $0.05,0.10$, and 0.15 respectively). Values of other parameters used in the simulations are listed in Table 1.

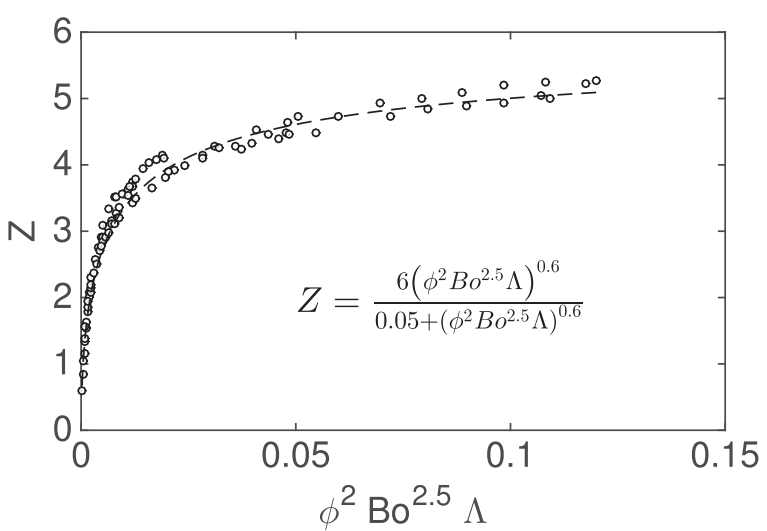

Fig. 9. Average liquid bridge coordination number vs. $\phi^{2} B 0^{2.5} \Lambda$ where $Z$ is approximated with the displayed fit. Values of parameters used in the simulations are listed in Table 1.

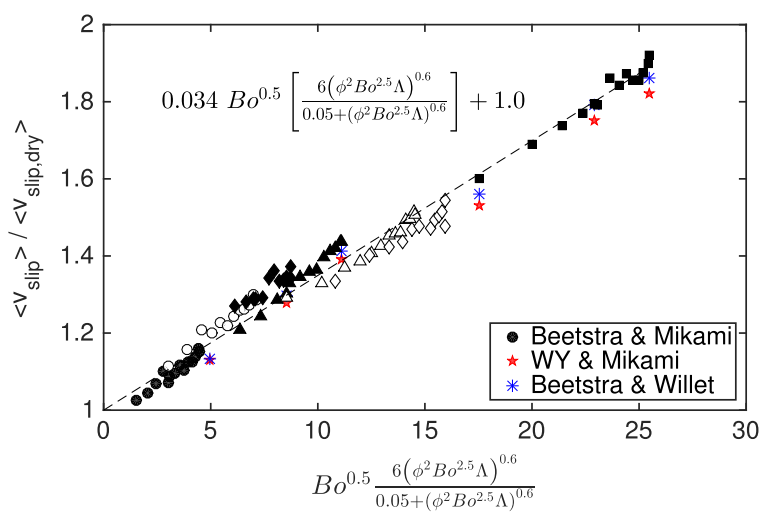

Fig. 10. Domain-averaged slip velocity vs. $B 0^{0.5} Z$, where $Z$ is approximated with a fit. The data shown is collected from fluidized systems modeled with three different sets of drag and liquid bridge force models: (i) The drag force of Beetstra et al. (2007) and liquid bridge force of Mikami et al. (1998), which served as the default models for data collection. The symbols here are the same as thosse used in Fig. 8. (ii) The drag force of Wen and Yu (1966) and liquid bridge force of Mikami et al. (1998). (iii) The drag force of Beetstra et al. (2007) and the liquid bridge force of Willett et al. (2000). Values of other parameters used in the simulations are listed in Table 1.

now replaced by the expression deduced from Fig. 9. The correlation shown in Fig. 10 captures the scaled slip velocity results reasonably well. Fig. 10 also includes results of simulations in

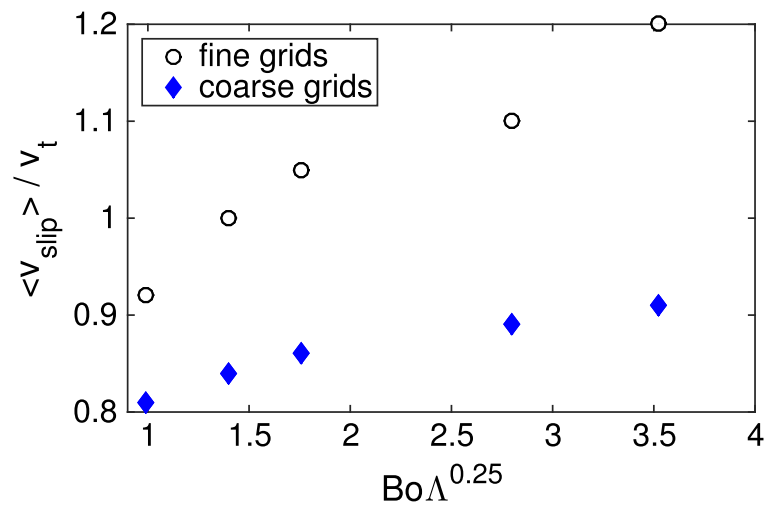

Fig. 11. Domain-averaged slip velocity for various wetting parameters with microscale (grid length $=3.07 d_{p}$ ) and coarse (grid length $=9.21 d_{p}$ ) grids. Solid volume fraction in the system is 0.15 and the remaining simulation parameters are given in Table 1.

which the drag model (Beetstra et al., 2007 to Wen and Yu, 1966) and liquid bridge force model (Mikami et al., 1998 to Willett et al., 2000) have been altered, displaying that the relation between fluidization behavior and the proposed correlation is robust to changes in the drag and liquid bridge force models employed.

While we began with a preliminary collapse in terms of $B o \Lambda^{n}$, Figs. 8 and 9 (and the correlations shown there) provide a more comprehensive collapse of our results, as illustrated in Fig. 10. One can easily recover the $B o \Lambda^{n} ; n=0.25 \pm 0.05$ dependence at fixed particle volume fractions: for small abscissa values, the results in Fig. 9 suggest that $Z \sim\left(\phi^{2} B o^{2.5} \Lambda\right)^{0.6}$ and so $B o^{0.5} Z$ scales as $\phi^{1.2}\left(B o \Lambda^{0.3}\right)^{2}$.

\subsection{Filtered drag coefficients}

Fig. 11 presents the domain-averaged slip velocities obtained for several different wet systems. The open circles are obtained in fine-grid simulations, while the solid diamonds correspond to grids that are three times larger in length in each direction. As all the particles are tracked in both cases, this difference is purely due to the fluid grid size. It is clear that the fluid grid size changes the results substantially, as finer fluid grids resolve the fluid flow field more fully. As illustrated in Appendix B, the fine grid results are essentially independent of fluid grid size. The inaccuracy in the coarse fluid grid can therefore be attributed to sub-grid scale fluid flow structures. Increasing the size of the coarse grid further only increases the disparity in predicted flow behavior. We then ask whether a suitably revised filtered drag coefficient (Igci et al., 2008,2011 ) could offer prediction closer to the fine-grid simulation results. (It should be emphasized that in this line of inquiry, we are only assessing the consequence of coarser fluid grids while exactly the same number of particles are simulated. If one coarsens the particle simulation as well, as in parcel-based simulations (Radl and Sundaresan, 2014), then additional modifications may be necessary; but it is not the focus of the present study.)

Filtered drag coefficients for wet systems are functions of filtered solid volume fraction, filter length, and wetting properties. Fig. 12 shows how the filtered drag coefficient changes with filter size for a range of filtered solid volume fractions in a dry and wet system ( $B o=25, \Lambda=0.0015)$. Here, the filtered drag coefficient is scaled with the microscopic drag coefficient obtained from the Beetstra drag model (Beetstra et al., 2007) with filtered slip velocity and filtered solid volume fraction. As the filter size increases, the filtered drag coefficient decreases to account for the reduced drag force experienced by the clusters and agglomerates. Fig. 12 suggests that as the filter size continues to increase, the drop in filtered drag coefficient becomes less pronounced. Fig. 13 shows how the scaled 
a

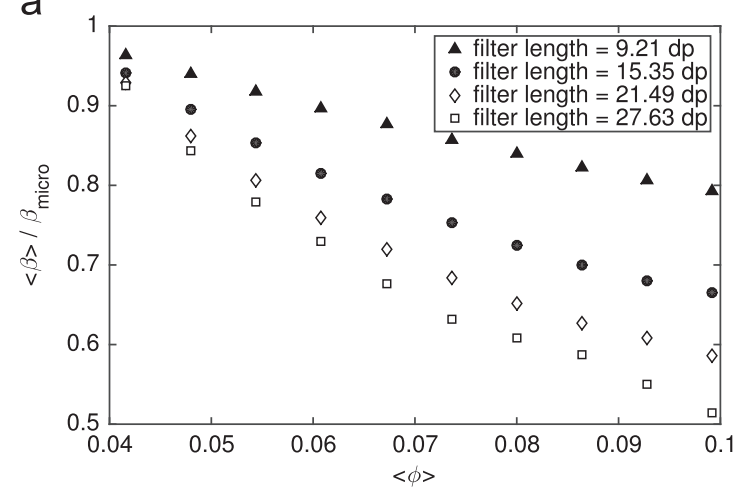

b

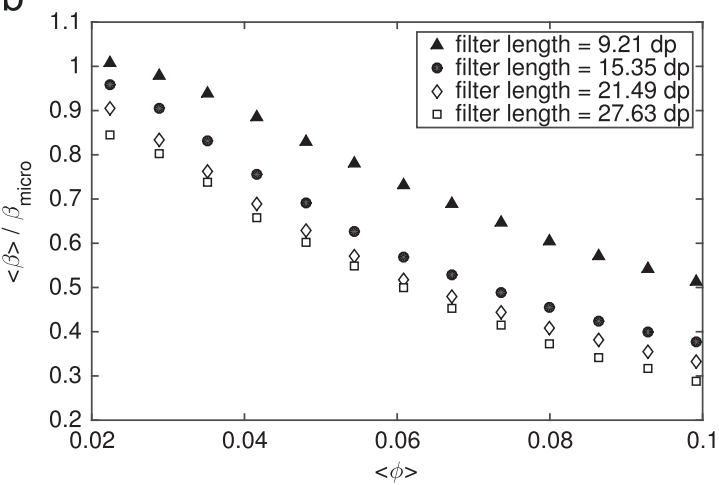

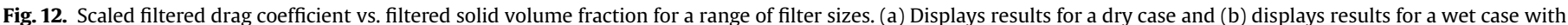
$B o=25, \Lambda=0.0015$. Solid volume fraction in the domain is 0.05 and the remaining simulation parameters are given in Table 1 .

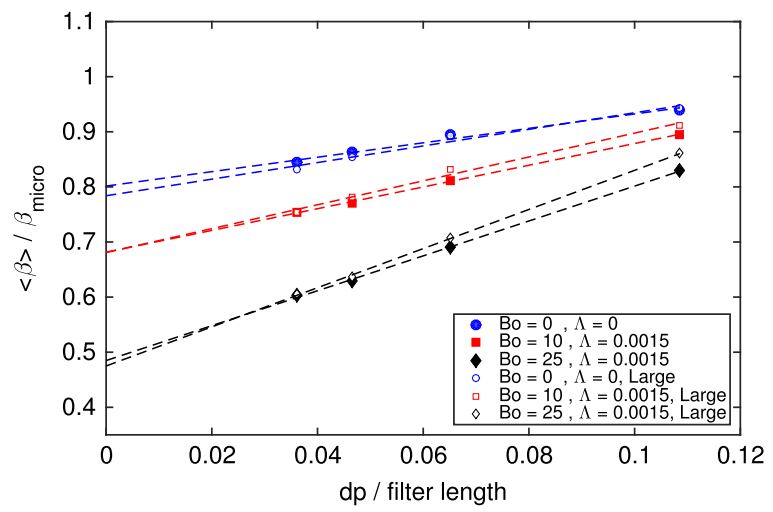

Fig. 13. Scaled filtered drag coefficient vs. inverse filter size for three systems of various liquid loading (a dry case and two wet cases with $B o=10$ and 25). Domainaveraged solid volume fraction and filtered solid volume fraction is 0.05 . Closed symbols represent results from a default domain size system of $18 \times 72 \times 18$ fluid grids, while open symbols represent results from a larger domain size system of $27 \times 108 \times 27$ fluid grids. Remaining simulation parameters are given in Table 1. Dashed lines are a linear fit to the data, extrapolated to infinite filter size.

filtered drag coefficient varies with inverse filter length for a dry case and two wet cases. For filter lengths three times the microscale length and greater, the filtered drag coefficient values appear to be linearly dependent on inverse filter length (see fit).

A somewhat different dependency of the filtered drag coefficient from the filter size was used in our previous work (Radl and Sundaresan, 2014). The dependency used by Radl and Sundaresan (2014) accounts for the effect of particle concentration and filter size, and approaches a linear dependency for the limit $d_{p} /$ (filter size) $\rightarrow 0$, as well as $\langle\beta\rangle / \beta_{\text {micro }} \rightarrow 1$ for a filter size of zero. The former limit is in line with the linear fit of data from comparably large filter sizes in our present contribution. Since we are mainly interested in the behavior for large filter sizes in the present work, we have not attempted to establish a more rigorous function modeling the filtered drag coefficient for small filter sizes.

This figure also shows the results for a domain that is 3.4 times larger in volume than the original domain (with same aspect ratio). It is clear that the results are only weakly dependent on the domain size. Even though it is questionable to extrapolate the results to "very large" filter sizes, it is useful to define a limiting value for the filtered drag coefficient, $\beta_{\Delta \infty}$, by extrapolating the lines to infinite filter size as indicated by the dashed lines in Fig. 13.

$\beta_{\Delta \infty}$ for a range of filtered solid volume fractions is shown in Fig. 14. As expected, increasing cohesion in the system, either through Bond number or liquid loading, decreases $\beta_{\Delta \infty}$. Fig. 15 displays $\beta_{\Delta \infty}$ scaled with its dry counterpart vs. solid volume

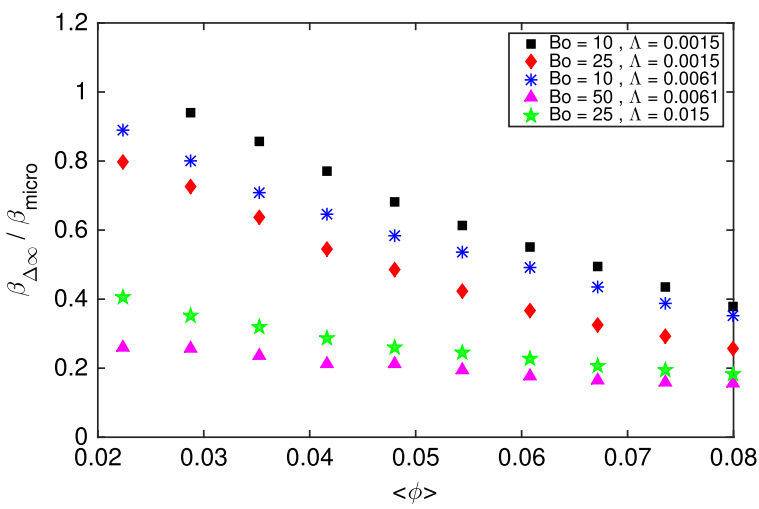

Fig. 14. Scaled filtered drag coefficient at infinite filter size limit vs. filtered solid volume fraction for a range of Bond numbers and liquid loading levels. Solid volume fraction in the domain is 0.05 . Remaining simulation parameters are given in Table 1.

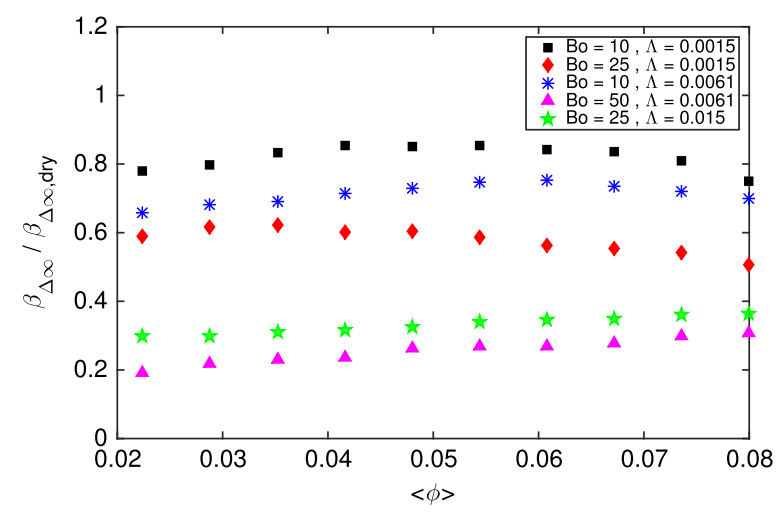

Fig. 15. Scaled filtered drag coefficient at infinite filter size limit vs. filtered solid volume fraction for a range of Bond numbers and liquid loading levels. $\beta_{\Delta \infty}$ is shown with a scaling of $\beta_{\mathrm{dry}}$. Solid volume fraction in the domain is 0.05 . Remaining simulation parameters are given in Table 1.

fraction for a number of wet cases. $\beta_{\Delta \infty}$, when scaled by $\beta_{\Delta \infty \text {,dry }}$ varies only weakly with filtered solid volume fraction.

The variation of $\beta_{\Delta \infty}$ with Bo is presented in Fig. 16a for three different liquid loading levels. In Fig. 16b, these results are collapsed in a manner analogous to what was done in Fig. $3 \mathrm{~b}$. This collapse suggests that the effect of wetness on the filtered drag coefficient could be quantified, at least as a start, in terms of the modified Bond number.

Fig. 17 provides the wet filtered drag coefficient as a function of the modified Bond number for a range of filter sizes. The results 
a

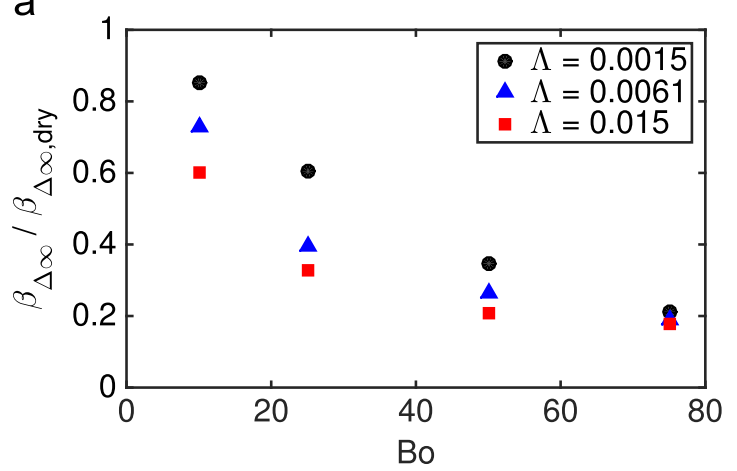

b

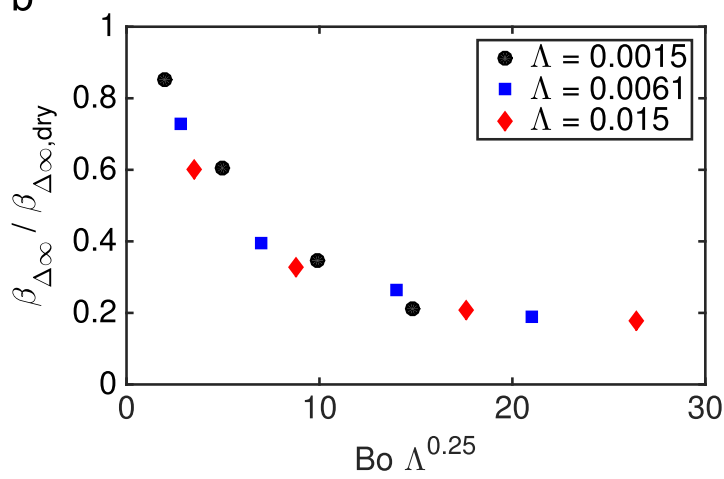

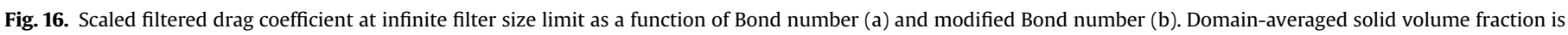
0.05 , and filtered solid volume fraction is 0.05 . Values of other parameters used in the simulations are listed in Table 1 .

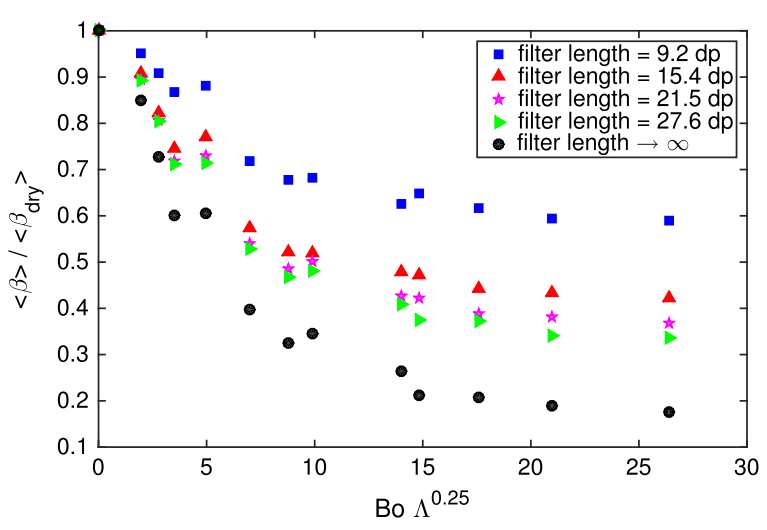

Fig. 17. Scaled filtered drag coefficient as a function of the modified Bond number. Results are shown for a range of filter sizes, including the infinite limit. Solid volume fraction in the domain is 0.05 , and the filtered solid volume fraction is 0.05 . Remaining simulation parameters are given in Table 1.

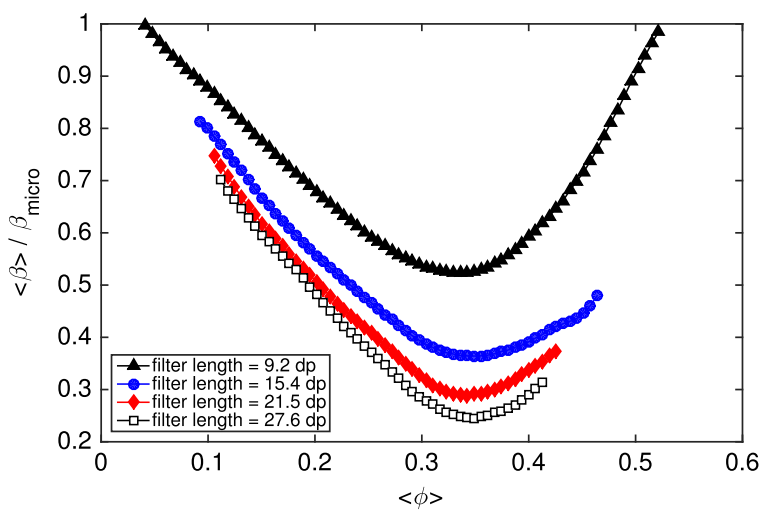

Fig. 18. Scaled dry filtered drag coefficient over a range of filtered solid volume fractions. Results shown are obtained by averaging over all dry simulations. Remaining simulation parameters are given in Table 1.

are shown for domain-averaged and filtered solid volume fractions of 0.05 . As expected, the wet filtered drag coefficient scaled with the corresponding dry system decreases with increasing filter size as well as $B o \Lambda^{0.25}$.

\subsection{Simulation with a coarser fluid grid}

It has already been noted by Radl and Sundaresan (2014) that correction to the drag coefficient is essential for dry systems if one hopes to achieve nearly the same domain-average slip velocity (which is a measure of macroscale fluidization characteristic in our test problem). The present study demonstrates that the need for

\section{Table 2}

Comparison of domain-averaged slip velocity between cases with varying grid size and drag laws. Grids sizes: fine (3.07 $\left.d_{p}\right)$ and coarse $\left(9.21 d_{p}\right)$. Drag laws: microscale $\left(\beta_{\text {micro }}\right)$, dry filtered $\left(\left\langle\beta_{\text {dry }}\right\rangle\right)$, and wet filtered $\left(\left\langle\beta_{\text {wet }}\right\rangle\right)$. The rightmost column entries designate a dry system utilizing a wet filtered drag coefficient. Volume fraction of particles in the domain is 0.05 . Values of other parameters used in the simulations are listed in Table 1.

\begin{tabular}{|c|c|c|c|c|c|c|c|}
\hline \multirow[t]{3}{*}{ Bo $\Lambda^{0.25}$} & \multirow[t]{3}{*}{ Bo } & \multirow[t]{3}{*}{$\Lambda$} & \multicolumn{5}{|l|}{$\left\langle v_{\text {slip }}\right\rangle / v_{t}$} \\
\hline & & & \multirow{2}{*}{$\begin{array}{l}\text { Fine grids } \\
\beta_{\text {micro }}\end{array}$} & \multicolumn{4}{|c|}{ Coarse grids } \\
\hline & & & & $\beta_{\text {micro }}$ & $\left\langle\beta_{\text {dry }}\right\rangle$ & $\left\langle\beta_{\text {wet }}\right\rangle$ & $\left\langle\beta_{\text {wet }}\right\rangle$ (dry system) \\
\hline 0.98 & 5 & 0.0015 & 0.96 & 0.94 & 0.98 & 0.99 & 0.93 \\
\hline 1.40 & 5 & 0.0061 & 1.01 & 1.01 & 1.01 & 1.05 & 0.95 \\
\hline 1.75 & 5 & 0.015 & 1.08 & 1.02 & 1.06 & 1.13 & 0.99 \\
\hline 2.79 & 10 & 0.0061 & 1.23 & 1.11 & 1.14 & 1.23 & 1.00 \\
\hline 3.50 & 10 & 0.015 & 1.35 & 1.15 & 1.18 & 1.32 & 1.05 \\
\hline
\end{tabular}

such a correction is more acute in wet systems. We did not attempt to formulate a filtered drag coefficient model for the wet system in the present study, as it seems sensible to work with larger systems than we considered here to obtain robust models. However, we did embark on a limited effort to assess the outcome of simulations with coarse fluid grids when coupled with a tentative formulation of filtered drag coefficients, as described below.

The dry filtered drag coefficient, for a range of filter sizes, is shown in Fig. 18. In what follows, we consider simulations that are analogous to the fine-grid simulations, except that we now use fluid grids that are three times larger in size (namely $9.2 d_{p}$ ). A functional form for the dry filtered drag coefficient is obtained by collecting filtered results from a number of simulations with solid loading varying from 0.05 to 0.50 . These results, for a filter length of $9.2 d_{p}$, are well captured by $1-1.2 \phi-6.6 \phi^{2}+17 \phi^{3}$.

Although the ratio $\left\langle\beta_{\text {wet }}\right\rangle /\left\langle\beta_{\text {dry }}\right\rangle$ shows some dependence on $\langle\phi\rangle$, for this exploratory study, we approximated it as a constant independent of $\langle\phi\rangle$, estimated by fitting the data in Fig. 17 for that filter size. We then performed fluidization simulations for two different $\langle\phi\rangle$ and several Bo and $\Lambda$ values; in each case we considered five different types of simulations:

1. Fine fluid grid simulations (with grid size $=3.07 d_{p}$ ) and microscopic drag law with no correction, with liquid bridge force included in the DEM simulations (referred to as "fine grids; $\beta_{\text {micro }}$ in Tables 2 and 3 ).

2. Coarse fluid grid simulations (with grid size $=9.21 d_{p}$ ) and microscopic drag law with no correction, with liquid bridge force included in the DEM simulations (referred to as "coarse grids; $\beta_{\text {micro }}$ in Tables 2 and 3 ). 
Table 3

Comparison of domain-averaged slip velocity between cases with varying grid size and drag laws. Grids sizes: fine (3.07 $\left.d_{p}\right)$ and coarse $\left(9.21 d_{p}\right)$. Drag laws: microscale $\left(\beta_{\text {micro }}\right)$, dry filtered $\left(\left\langle\beta_{\text {dry }}\right\rangle\right)$, and wet filtered $\left(\left\langle\beta_{\text {wet }}\right\rangle\right)$. The rightmost column entries designate a dry system utilizing a wet filtered drag coefficient. Volume fraction of particles in the domain is 0.15 . Values of other parameters used in the simulations are listed in Table 1.

\begin{tabular}{|c|c|c|c|c|c|c|c|}
\hline \multirow{3}{*}{ Bo $\Lambda^{0.25}$} & \multirow{3}{*}{ Bo } & \multirow{3}{*}{$\Lambda$} & \multicolumn{5}{|l|}{$\left\langle v_{\text {slip }}\right\rangle / v_{t}$} \\
\hline & & & \multirow{2}{*}{$\begin{array}{l}\text { Fine grids } \\
\beta_{\text {micro }}\end{array}$} & \multicolumn{4}{|c|}{ Coarse grids } \\
\hline & & & & $\beta_{\text {micro }}$ & $\left\langle\beta_{\text {dry }}\right\rangle$ & $\left\langle\beta_{\text {wet }}\right\rangle$ & $\left\langle\beta_{\text {wet }}\right\rangle$ (dry system) \\
\hline 0.98 & 5 & 0.0015 & 0.92 & 0.81 & 0.95 & 0.97 & 0.87 \\
\hline 1.40 & 5 & 0.0061 & 1.00 & 0.84 & 1.01 & 1.06 & 0.87 \\
\hline 1.75 & 5 & 0.015 & 1.05 & 0.86 & 1.04 & 1.08 & 0.91 \\
\hline 2.79 & 10 & 0.0061 & 1.10 & 0.89 & 1.06 & 1.13 & 0.94 \\
\hline 3.50 & 10 & 0.015 & 1.20 & 0.91 & 1.13 & 1.14 & 0.97 \\
\hline
\end{tabular}

3. Coarse fluid grid simulations (with grid size $=9.21 d_{p}$ ) and filtered drag coefficient determined for dry systems for a filter size equal to the coarse grid size, with liquid bridge force included in the DEM simulations (referred to as "coarse grids; $\left\langle\beta_{\text {dry }}\right\rangle$ " in Tables 2 and 3).

4. Coarse fluid grid simulations (with grid size $=9.21 d_{p}$ ) and filtered drag coefficient determined for wet systems for a filter size equal to the coarse grid size, with liquid bridge force included in the DEM simulations (referred to as "coarse grids; $\left\langle\beta_{\text {wet }}\right\rangle$ " in Tables 2 and 3).

5. Coarse fluid grid simulations (with grid size $=9.21 d_{p}$ ) and filtered drag coefficient determined for wet systems for a filter size equal to the coarse grid size, ignoring the liquid bridge force present in the DEM simulations (referred to as "coarse grids; $\left\langle\beta_{\text {wet }}\right\rangle$ (dry system)" in Tables 2 and 3 ).

The results from these simulations are as follows: For low values of $B o \Lambda^{0.25}$ and $\phi=0.05$, there are small differences between the results for the various cases. Yet, as solid volume fraction or cohesion increases, coarse simulations with no corrections show marked deficiency in accuracy (compare columns 4 and 5). Simulations employing a wet filtered drag coefficient and ignoring liquid bridges completely are no better (compare columns 4 and 8). In some instances, there is little difference between applying the wet filtered drag or dry filtered drag corrections (compare columns 6 and 7), but in other cases, they differ appreciably. On the whole, column 7 is closest to column 4 , suggesting that when coarse fluid grids are employed, it is preferable to use the filtered drag coefficient determined for wet systems.

As a final remark, we note that, in the periodic domain simulations performed in this study, the domain-averaged slip velocity changes with domain size. This certainly indicates that this coarse metric is affected by the imposed periodicity, which curbs the extent of inhomogeneity that could form. As a result, it is natural to question whether the filtered drag coefficients would differ if one employs very large domains. A definitive answer to this question will come only through very large scale simulations, which are beyond what our resources allow. The intent of the present study is to expose the trends and make an initial assessment of the outcome of coarse-graining wet and dry fluidization results. In our opinion, the conclusion that it is best to employ filtered drag coefficients determined for wet systems when performing CFD-DEM simulations of wet systems with coarse fluid grids should not be affected by this periodicity limitation.

\section{Summary}

We have investigated through CFD-DEM simulations the fluidization behavior of wet particles in small periodic domains. Our simulations took into account only the surface tension force of attraction induced by the liquid bridge between particles. In order to keep the analysis simple, it was postulated that collisions between two particles resulted in the formation of a liquid bridge of specified mass. Simulations were carried out for different particle fractions $(\phi)$, liquid loadings $(\Lambda)$, and Bond numbers $(B o)$. Our simulations typically began with a homogeneous distribution of particles in the domain with no liquid bridges; inhomogeneity developed over a period of time as a result of inherent instability associated with fluidization, as well as particle agglomeration induced by liquid bridge cohesion, eventually reaching a statistical steady state. As one would expect, an increase in the strength of cohesion leads to the formation of larger agglomerates and an increase in the average liquid-bridge coordination number $(Z)$, and these are accompanied by an increase in the domain-averaged gas-particle slip velocity required to fully support the particles through fluidization. At sufficiently large strength of cohesion the agglomerate size can become as large as that of the domain and the domain-averaged slip velocity was found to saturate. Such a plateau is likely due to the finite size of the simulation domain. With this in mind, we excluded from our analysis the results in the plateau region. It is found that the scaled domain-averaged slip velocity increases linearly with $B 0^{0.5} Z$. Furthermore, $Z$ could be correlated as a function of $\phi^{2} B 0^{2.5} \Lambda$. Combining these two, a simple correlation for the scaled domain-averaged slip velocity is deduced in terms of $\phi, B o$, and $\Lambda$. This exercise revealed that Bo and $\Lambda$ could be combined into a modified Bond number defined as $B o \Lambda^{n} ; n=0.25 \pm 0.05$.

Filtered drag coefficients, determined by systematically coarsegraining the results from fine-grid simulations, showed an inverse dependence on filter size, while also decreasing with increasing modified Bond number. Test simulations comparing the prediction of coarse and fine fluid grid CFD-DEM simulations revealed that coarse CFD-DEM simulations employing the wet filtered drag coefficient more closely matched fine-grid results than their counterparts that included no correction to the drag law.

Future studies should address the presence of an inter-particle force due to a viscous resistance of the liquid bridge, further promoting agglomeration in the wet gas-solid fluidized system. Additionally, the simulations can be further refined by allowing for a finite rate of liquid bridge filling. While a methodology was provided for the coarsening of the fluid grid, coarsening of the particle phase is also required for large scale simulations. Particle coarsening methods are a natural next step in the work and can take advantage of the filtered drag coefficient provided here. These are worthy of further investigation and will contribute to better mesoscopic models for the hydrodynamics of wet fluidized beds.

\section{Nomenclature}

Latin letters
$B o=\frac{6 \gamma}{d_{p}^{2} \rho_{p} g}$
$C a=\frac{\mu_{1} v_{t}}{\gamma}$
$d_{p}$
$e_{\mathrm{pp}}$
$\mathbf{f}_{b, i k}$
$\mathbf{f}_{c o n t, i j}^{n}$
$\mathbf{f}_{c o n t, i j}^{t}$
$\mathbf{f}_{d}$

Bond number (-)

capillary number (-)

particle diameter $(\mathrm{m})$

restitution coefficient of the dry particles $(-)$

liquid bridge force between particles $(\mathrm{N})$

normal contact force between particles $(\mathrm{N})$

tangential contact force between particles $(\mathrm{N})$ 


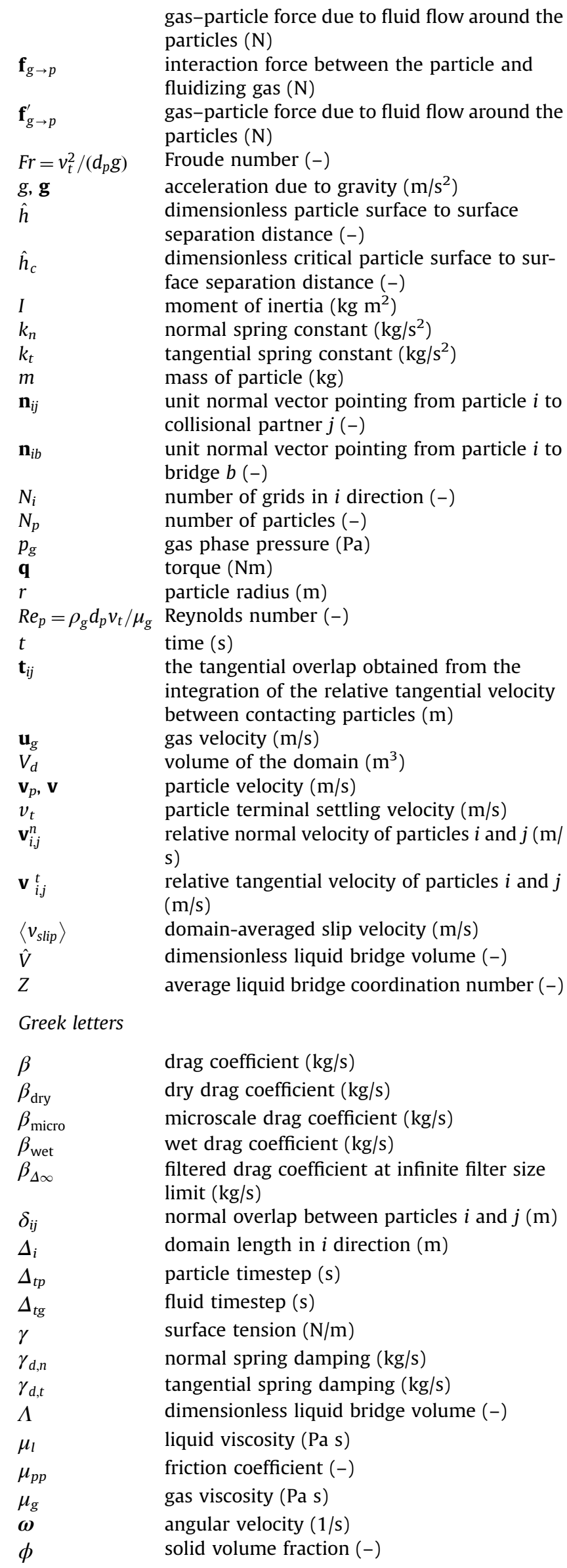

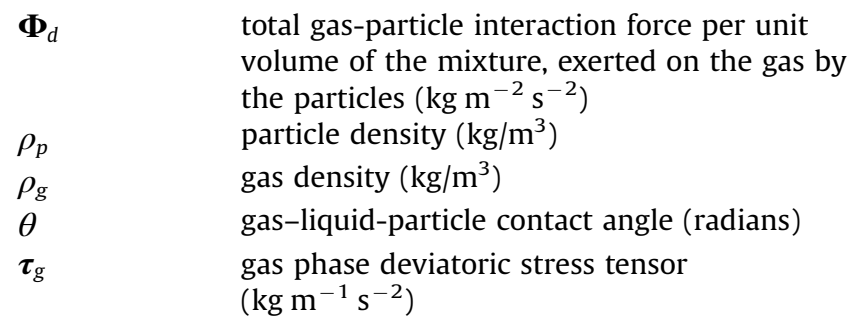

\section{Acknowledgments}

Financial support for this work was provided by Syncrude, Canada. We gratefully acknowledge any helpful discussions with Michael Wormsbecker, Jennifer McMillan, and Kevin Reid of Syncrude.

Stefan Radl acknowledges support through Project J-3072 sponsored by the Austrian Science Foundation (www.fwf.ac.at).

\section{Appendix A. Liquid transfer model}

A constant liquid bridge volume (CLBV) model is employed in this study, in which the volume of the liquid bridge that is formed upon particle-particle contact is set as an input to the simulation. This approach, while popular in the literature (Mikami et al., 1998; Radl et al., 2010), is only an approximation of what occurs in a real system. A more accurate representation of the physical system would be to let the volume of liquid occupying the bridge be determined upon collision, rather than specified as an input into the simulation. We performed a limited study, allowing for such a liquid transfer (LT) model, where we specify the total amount of liquid in the system as an input. As an initial condition, the liquid is uniformly distributed on all of the particles. When two particles collide, the particles supply a portion of free liquid $\left(V_{l p, i}\right)$ to the bridge. This portion of liquid is provided by the model of Shi and McCarthy (2008):

$V_{l b}=\frac{1}{2}\left(V_{l p, i}+V_{l p, j}\right)\left(1-\frac{\sqrt{3}}{2}\right)$

When a liquid bridge connecting particles $i$ and $j$ ruptures, the liquid in the bridges is given to the two particles equally and added to the free liquid on the particle. Both the free liquid on the particle surface and the liquid in the bridges are tracked during simulation. In such simulations, due to the transfer of free liquid from the particle surface to the bridge, the inventory of free liquid on the particle will not be identical among particles. Furthermore, the volume of liquid in the bridges results in a distribution.

The distribution of liquid in the bridges follows a normal distribution, as displayed in Fig. A1a. Thus, for any given system, the mean liquid volume on the particles provides a value for $\Lambda$. Such $\Lambda$ values may then be collected from a range of systems and used as an input into simulations that utilize the constant liquid bridge volume model. Fig. A1b compares the domain-averaged slip velocity for two sets of simulations: a set utilizing a constant liquid bridge volume model, and a set utilizing a liquid transfer model. Little to no change in fluidization behavior is observed as the liquid bridges are modeled with a constant liquid bridge volume or liquid transfer model, suggesting that the constant liquid bridge volume model may be used in place of the more advanced liquid transfer model to predict flow behavior in homogeneously wetted systems. 
a

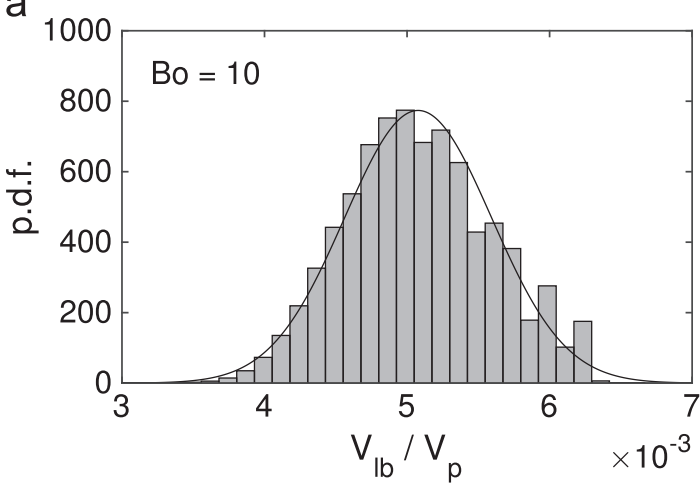

b

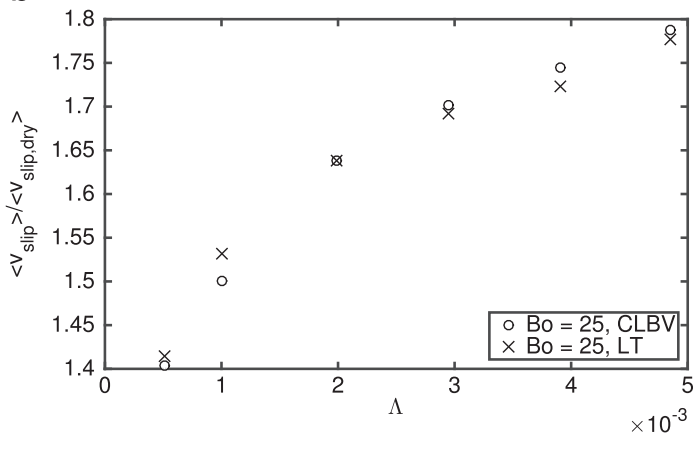

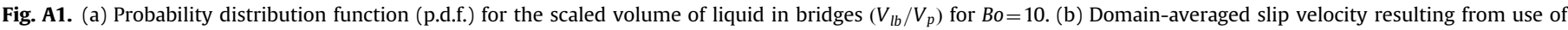

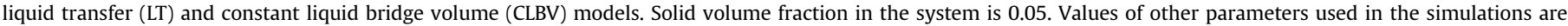
listed in Table 1.

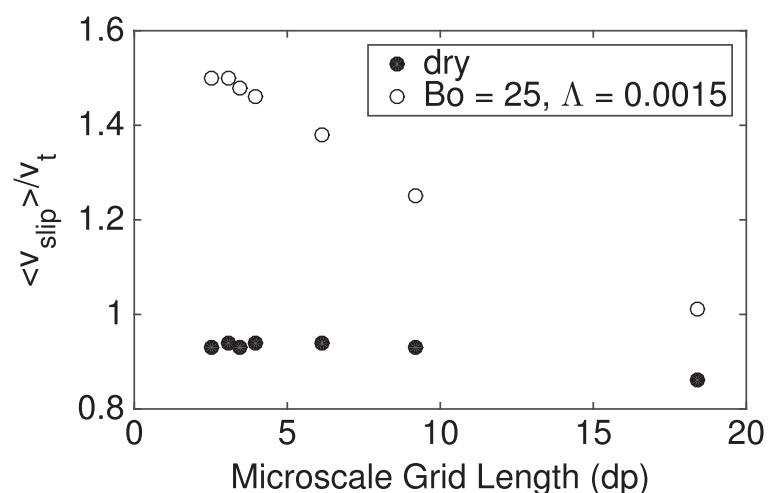

Fig. B1. Domain-averaged slip velocity for various microscale grid sizes. Solid volume fraction in the system is 0.05 and the remaining simulation parameters are given in Table 1.

\section{Appendix B. Determination of microscale grid size}

In performing Euler-Lagrange simulations, a computational grid size for the fluid cells must be chosen (as it is a simulation input). Clearly, no fluid flow behavior on length scales smaller than the computational grid size is resolved in a simulation. Thus, while large grid sizes allow for quicker computations, they may yield inaccurate results. In order to select the proper microscale grid length for use in this study, a series of simulations are performed in which the computational grid size is varied and the flow behavior is compared. The flow behavior itself is quantitatively measured using the domain-averaged slip velocity (as discussed in Section 2.3).

The Euler-Lagrange simulations used in the study utilize a periodic domain with flow driven by a pressure gradient that balances the weight of the system. Parameter values used in the simulations are given in Table 1 , with the exception of the fluid grid size which was varied. Two systems of solid volume fraction 0.05 were tested, a wet system with $B o=25$ and $\Lambda=0.0015$, and a corresponding dry system. Fig. B1 shows the domain-averaged slip velocity corresponding to various fluid grid sizes for both the dry and wet case. The wet case displays a much larger grid size dependence than the dry case. In both cases, the domain-averaged slip velocity is nearly independent of grid size for grid size $\sim 3 d_{p}$. Based on this consideration, we have adopted the fluid grid size shown in Table 1 for all simulations.

\section{References}

Adams, M., Perchard, V., 1985. The cohesive forces between particles with interstitial fluid. In: Institution of Chemical Engineering Symposium, pp. 147-160.
Agrawal, K., Loezos, P.N., Syamlal, M., Sundaresan, S., 2001. The role of meso-scale structures in rapid gas-solid flows. J. Fluid Mech. 445 (October), 151-185.

Anand, A., Curtis, J.S., Wassgren, C.R., Hancock, B.C., Ketterhagen, W.R., 2009. Predicting discharge dynamics of wet cohesive particles from a rectangular hopper using the discrete element method (DEM). Chem. Eng. Sci. 64 (December), 5268-5275.

Anand, A., Curtis, J.S., Wassgren, C.R., Hancock, B.C., Ketterhagen, W.R., 2010. Segregation of cohesive granular materials during discharge from a rectangular hopper. Granul. Matter 12 (March), 193-200.

Antonyuk, S., Heinrich, S., Deen, N., Kuipers, H., 2009. Influence of liquid layers on energy absorption during particle impact. Particuology 7, 245-259.

Arbel, A., Huang, Z., Rinard, I.H., Shinnar, R., Sapre, A.V., 1995. Dynamic and control of fluidized catalytic crackers. 1. Modeling of the current generation of FCC's. Ind. Eng. Chem. Res. 34 (April), 1228-1243.

Beetstra, R., van der Hoef, M.A., Kuipers, J.A.M., 2007. Drag force of intermediate Reynolds number flow past mono- and bidisperse arrays of spheres. AIChE J. 53 (2), 489-501.

Capecelatro, J., Desjardins, O., Fox, R.O., 2014. Numerical study of collisional particle dynamics in cluster-induced turbulence. J. Fluid Mech. 747 (April), R2.

Christensen, F., Bertelsen, P., 1997. Qualitative description of the Wurster-based fluid-bed coating process. Drug Dev. Ind. Pharm. 23 (5), 451-463.

Cundall, P.A., Strack, O.D.L., 1979. A discrete numerical model for granular assemblies. Géotechnique 29 (January), 47-65.

D'Amore, M., Donsi, G., Massimilla, L., 1979. The influence of bed moisture on fluidization characteristics of fine powders. Powder Technol. 23, 253-259.

Darabi, P., Pougatch, K., Salcudean, M., Grecov, D., 2009. A novel coalescence model for binary collision of identical wet particles. Chem. Eng. Sci. 64 (April), 1868-1876.

Darabi, P., Li, T., Pougatch, K., Salcudean, M., Grecov, D., 2010. Modeling the evolution and rupture of stretching pendular liquid bridges. Chem. Eng. Sci. 65 (August), 4472-4483.

Darabi, P., Pougatch, K., Salcudean, M., Grecov, D., 2010. Agglomeration of Bitumencoated coke particles in fluid cokers. Int. J. Chem. Reactor Eng. 8 (A122).

Darabi, P., Pougatch, K., Salcudean, M., Grecov, D., 2011. DEM investigations of fluidized beds in the presence of liquid coating. Powder Technol. 214 (December), 365-374.

Deen, N., Van Sint Annaland, M., Van der Hoef, M., Kuipers, J., 2007. Review of discrete particle modeling of fluidized beds. Chem. Eng. Sci. 62 (January), $28-44$.

Donahue, C., Hrenya, C., Davis, R., 2010. Stokes's cradle: Newton's cradle with liquid coating. Phys. Rev. Lett. 105 (July), 1-2.

Fox, R.O., 2012. Large-Eddy-simulation tools for multiphase flows. Annu. Rev. Fluid Mech. 44 (January), 47-76.

Fries, L., Antonyuk, S., Heinrich, S., Palzer, S., 2011. DEM-CFD modeling of a fluidized bed spray granulator. Chem. Eng. Sci. 66, 2340-2355.

Fries, L., Antonyuk, S., Heinrich, S., Dopfer, D., Palzer, S., 2013. Collision dynamics in fluidized bed granulators: a DEM-CFD study. Chem. Eng. Sci. 86, 108-123.

Galvin, J.E., Benyahia, S., 2014. The effect of cohesive forces on the fluidization of aeratable powders. AIChE J. 60 (2), 473-484.

Gidaspow, D., 1994. Multiphase Flow and Fluidization: Continuum and Kinetic Theory Descriptions. Academic Press, San Diego.

Glasser, B., Sundaresan, S., Kevrekidis, I., 1998. From bubbles to clusters in fluidized beds. Phys. Rev. Lett. 81 (August), 1849-1852.

Gray, M.R., 2002. Fundamentals of bitumen coking processes analogous to granulations: a critical review. Can. J. Chem. Eng. 80 (June), 393-401.

Hapgood, K., Iveson, S., Liu, L., 2007. Handbook of Powder Technology, vol. 11 ed.

Herminghaus, S., 2005. Dynamics of wet granular matter. Adv. Phys. 54 (May), $221-261$.

Hou, Q., Zhou, Z., Yu, A., 2012. Micromechanical modeling and analysis of different flow regimes in gas fluidization. Chem. Eng. Sci. 84 (December), 449-468.

Igci, Y., Andrews, A.T., Sundaresan, S., O'Brien, T., 2008. Filtered two-fluid models for fluidized gas-particle suspensions. AIChE J. 54 (6), 1431-1448. 
Igci, Y., Pannala, S., Benyahia, S., Sundaresan, S., June 2011. Validation studies on filtered model equations for gas-particle flows in risers. Ind. Eng. Chem. Res.

Jackson, R., 2000. The Dynamics of Fluidized Particles. Cambridge University Press, Cambridge, UK.

Kantak, A.A., Hrenya, C.M., Davis, R.H., 2009. Initial rates of aggregation for dilute, granular flows of wet particles. Phys. Fluids 21 (2), 13.

Li, J., Kuipers, J., 2005. On the origin of heterogeneous structure in dense gas-solid flows. Chem. Eng. Sci. 60 (March), 1251-1265.

Lian, G., Thornton, C., Adams, M.J., 1993. A theoretical study of the liquid bridge forces between two rigid spherical bodies. J. Colloid Interface Sci. 161 (November), 138-147.

Liu, P., Yang, R., Yu, a.B., 2013. DEM study of the transverse mixing of wet particles in rotating drums. Chem. Eng. Sci. 86 (February), 99-107.

Luding, S., 2008. Introduction to discrete element methods. Basic of contact force models and how to perform the micro-macro transition to continuum theory. Revue européenne de génie civil 12 (October), 785-826.

Maroglou, A., Nienow, A.W., 1985. Fluidized bed granulation: a procedure for determining its feasibility and suitable operating parameters. In: Institution of Chemical Engineers Symposium Series, vol. 91, pp. 15-25.

McLaughlin, L.J., Rhodes, M.J., 2001. Prediction of fluidized bed behavior in the presence of liquid bridges. Powder Technol. 114 (January), 213-223.

Mikami, T., Kamiya, H., Horio, M., 1998. Numerical simulation of cohesive powder behavior in a fluidized bed. Chem. Eng. Sci. 53 (10), 1927-1940.

Milioli, C.C., Milioli, F.E., Holloway, W., Agrawal, K., Sundaresan, S., 2013. Filtered two-fluid models of fluidized gas-particle flows: new constitutive relations. AIChE J. 59 (9), 3265-3275.

Mohagheghi, M., Hamidi, M., Briens, C., Berruti, F., McMillan, J., 2014. The effects of liquid properties and bed hydrodynamics on the distribution of liquid on solid fluidized particles in a cold-model fluidized bed. Powder Technol. 256 (April), $5-12$.

Muzzio, F.J., Shinbrot, T., Glasser, B.J., 2002. Powder technology in the pharmaceutical industry: the need to catch up fast. Powder Technol. 124 (April), 1-7.

Ozel, A., Fede, P., Simonin, O., 2013. Development of filtered Euler-Euler two-phase model for circulating fluidized bed: high resolution simulation, formulation and a priori analyses. Int. J. Multiph. Flow 55, 43-63.

Passos, M., Mujumdar, A., 2000. Effect of cohesive forces on fluidized and spouted beds of wet particles. Powder Technol. 110 (June), 222-238.

Pitois, O., Moucheront, P., Chateau, X., 2000. Liquid bridge between two moving spheres: an experimental study of viscosity effects. J. Colloid Interface Sci. 231 (December), 26-31.

Pitois, O., Moucheront, P., Chateau, X., 2001. Rupture energy of a pendular liquid bridge. Phys. J. B 86, 79-86.

Radl, S., Sundaresan, S., 2014. A drag model for filtered Euler-Lagrange simulations of clustered gas-particle suspensions. Chem. Eng. Sci. 117 (September), 416-425.

Radl, S., Kalvoda, E., Glasser, B.J., Khinast, J.G., 2010. Mixing characteristics of wet granular matter in a bladed mixer. Powder Technol. 200 (3), 171-189.

Rajniak, P., Stepanek, F., Dhanasekharan, K., Fan, R., Mancinelli, C., Chern, R., 2009. A combined experimental and computational study of wet granulation in a Wurster fluid bed granulator. Powder Technol. 189 (January), 190-201.
Rhodes, M.J., Wang, X.S., Nguyen, M., Steward, P., Liffman, K., 2001. Use of discrete element method simulation in studying fluidization characteristics: influence of interparticle force. Chem. Eng. Sci. 56, 69-76.

Seville, J.P.K., Clift, R., 1984. The effect of thin liquid layers on fluidization characteristics. Powder Technol. 37, 117-129.

Seville, J.P.K., 1987. Tribology in Particulate Technology.

Shelukar, S., 2000. Identification and characterization of factors controlling tablet coating uniformity in a Wurster coating process. Powder Technol. 110 (May) 29-36.

Shi, D., McCarthy, J., 2008. Numerical simulation of liquid transfer between particles. Powder Technol. 184 (May), 64-75.

Snider, D., 2001. an incompressible three-dimensional multiphase particle-in-cel model for dense particle flows. J. Comput. Phys. 170 (July), 523-549.

Sommerfeld, M., Wirth, K.-E., Muschelknautz, U. L3 two-phase gas-solid flow. In: VDI Heat Atlas, Second Edition, 2010, Springer, Berlin.

Sundaresan, S., 2000. Modeling the hydrodynamics of multiphase flow reactors: current status and challenges. AIChE J. 46 (June), 1102-1105.

Sundaresan, S., 2003. Instabilities in fluidized beds. Annu. Rev. Fluid Mech. 35 (January), 63-88.

Sutkar, V.S., Deen, N.G., Padding, J.T., Kuipers, J.A.M., Salikov, V., Cruger, B., Antonyuk, S., Heinrich, S., 2015. A novel approach to determine wet restitution coefficients through a unified correlation and energy analysis. AIChE J. 61 (3), 769-779.

Tenneti, S., Subramaniam, S., 2014. Particle-resolved direct numerical simulation for gas-solid flow model development. Annu. Rev. Fluid Mech. 46 (January), 199-230.

van der Hoef, M.A., Ye, M., van Sing Annaland, M., Andrews IV, A., Sundaresan, S. Kuipers, J., 2006. Multiscale modeling of gas-fluidized beds. Comput. Fluid Dyn. 31, 65-149.

van der Hoef, M., van Sint Annaland, M., Deen, N., Kuipers, J., 2008. Numerica simulation of dense gas-solid fluidized beds: a multiscale modeling strategy. Annu. Rev. Fluid Mech. 40 (January), 47-70.

Vincent, S., César, J., Brandle de Motta, J., Sarthou, A., Estivalezes, J.-l., Simonin, O. Climent, E., 2014. A Lagrangian VOF tensorial penalty method for the DNS of resolved particle-laden flows. J. Comput. Phys. 256, 582-614.

Wen, C., Yu, Y., 1966. Mechanics of fluidization. Chem. Eng. Prog. Symp. Ser. 62 (62), $100-111$.

Willett, C.D., Adams, M.J., Johnson, S.A., Seville, J.P.K., 2000. Capillary bridges between two spherical bodies. Langmuir 16 (November), 9396-9405.

Wormsbecker, M., Pugsley, T., 2008. The influence of moisture on the fluidization behavior of porous pharmaceutical granule. Chem. Eng. Sci. 63 (August), 4063-4069.

Zhou, Z.Y., Kuang, S.B., Chu, K.W., Yu, A.B., 2010. Discrete particle simulation of particle-fluid flow: model formulations and their applicability. J. Fluid Mech. 661 (August), 482-510.

Zhou, Y., Ren, C., Wang, J., Yang, Y., 2012. Characterization on hydrodynamic behavior in liquid-containing gas-solid fluidized bed reactor. Part. Technol Fluid. 59 (4), 1056-1065. 